\section{La Révolution française}

Cahiers de l'Institut d'histoire de la Révolution française

$3 \mid 2012$

Les comités des assemblées révolutionnaires : des laboratoires de la loi

\title{
Métamorphoses d'un comité : le Comité des pétitions et de correspondance sous la Convention nationale
}

\section{Maria Betlem Castellà i Pujols}

\section{OpenEdition}

\section{Journals}

Édition électronique

URL : http://journals.openedition.org//rf/702

DOI : $10.4000 /$ Irf.702

ISSN : 2105-2557

\section{Éditeur}

IHMC - Institut d'histoire moderne et contemporaine (UMR 8066)

Référence électronique

Maria Betlem Castellà i Pujols, « Métamorphoses d'un comité : le Comité des pétitions et de

correspondance sous la Convention nationale », La Révolution française [En ligne], 3 | 2012, mis en ligne le 20 décembre 2012, consulté le 30 avril 2019. URL : http://journals.openedition.org//rf/702 ; DOI : $10.4000 /$ Irf.702

Ce document a été généré automatiquement le 30 avril 2019.

(C) La Révolution française 


\title{
Métamorphoses d'un comité : le Comité des pétitions et de correspondance sous la Convention nationale ${ }^{1}$
}

\author{
Maria Betlem Castellà i Pujols
}

1 Le Comité des pétitions et de correspondance fut créé le 2 octobre $1792^{2}$, le même jour que la plupart des comités qui furent mis sur pied au tout début de la jeune République.

2 Seuls le Comité de la guerre et le Comité de constitution qui devait rédiger une nouvelle carte constitutionnelle avaient été créés quelques jours auparavant, le premier ${ }^{3}$, au lendemain de la proclamation de la République, le 23 septembre 1792, le deuxième ${ }^{4}$, une semaine après cet événement important. Les autres comités furent créés les $1^{\text {er }}$ et 2 octobre après que Constant-Joseph Eugène Gossuin, membre du Comité des pétitions de l'Assemblée législative depuis le 24 octobre 1791, eut présenté aux nouveaux députés la liste des comités existants sous l'Assemblée précédente ${ }^{5}$. Il fallait décider des comités que l'on devait supprimer et de ceux que l'on voulait maintenir.

3 La Convention nationale ne supprima aucun des comités qui restaient en fonctionnement en septembre 1792 ni aucune des commissions. Néanmoins, elle mena à terme une politique d'absorption et de regroupements. Le Comité féodal, pour citer un exemple, fut intégré dans le Comité de législation ${ }^{6}$, les commissaires Inspecteurs de la Salle formèrent un nouveau comité conjointement avec les inspecteurs du secrétariat et de l'imprimerie ${ }^{7}$, et la Commission extraordinaire des douze absorba le Comité de surveillance, donnant lieu au Comité de sûreté générale ${ }^{8}$. Dans ce contexte d'absorption et de regroupements, apparut le Comité des pétitions et de correspondance qui naquit de la décision de l'Assemblée d'ajouter au Comité des pétitions la commission de correspondance. 


\section{Origines. Le Comité des pétitions et la commission de correspondance de l'Assemblée législative}

4 Le Comité des pétitions s'était substitué au Comité des rapports et des recherches le 14 octobre 1791 pour assurer la continuité des travaux du Comité des rapports9. Il devait gérer, entre autres, la correspondance qui rendait compte des vœux, des espoirs, des plaintes, des pétitions et des demandes des commettants, qui n'étaient pas adressés auparavant à d'autres comités, ni communiqués à l'Assemblée par le Bureau des renvois et des correspondances sous la direction du président du Corps législatif. Étant donné que l'Assemblée législative n'avait pas le temps nécessaire pour se faire lire toute la correspondance qu'on lui adressait, le Comité des pétitions avait la compétence de décider des informations qu'elle devait connaitre parmi toutes celles qu'il recevait du Bureau des renvois et des correspondances. Le Comité des pétitions exerçait alors la fonction de filtre sur la correspondance reçue. Il décidait des informations qui devaient être communiquées ou non aux députés, de celles qui devaient être envoyées aux comités ou aux ministres, et de celles, enfin, qui pouvaient être rejetées. Il avait aussi le pouvoir d'écarter des informations. De fait, un certain nombre de lettres furent étiquetées avec la mention «Il n'y a pas lieu à délibérer $»^{10}$. À cet égard, le choix du Comité des pétitions pouvait répondre aux critères particuliers, politiques ou arbitraires de ses membres. À cause de cette compétence de filtrage de l'information, le Comité des pétitions pouvait orienter le débat parlementaire et influencer les députés du Corps législatif dans leur prise de décisions. Et cela parce que si les députés voulaient légiférer en tenant compte de la volonté de leurs commettants, ils devaient être au courant des informations transmises par le Comité des pétitions. Son travail fut aussi d'une importance remarquable du fait du volume de correspondance qu'il géra. De janvier à septembre 1792, le Comité des pétitions fut celui qui reçut le plus de correspondance enregistrée parmi tous les comités et commissions en place à cette époque-là ${ }^{11}$, par les secrétaires-commis du Bureau des renvois et des correspondances. Si le Comité des pétitions était né pendant les premiers jours de l'Assemblée législative, la commission de correspondance naquit au cours de ses dernières séances, une semaine après la chute de la monarchie ${ }^{12}$. D'autre part, si le Comité des pétitions fut chargé de recevoir, pour ainsi dire, la volonté générale, et d'être, comme le dit Gossuin, l'organe des corps administratifs et des citoyens de l'Empire auprès des députés (« Messieurs, c'est toujours avec une satisfaction nouvelle que votre Comité des Pétitions est auprès de vous l'organe des corps administratifs et des citoyens de l'Empire. $»^{13}$ ), la commission de correspondance fut l'organe de l'Assemblée, au milieu des corps administratifs et des citoyens de l'Empire, l'instrument par lequel l'Assemblée pouvait être présente partout. Dès le 17 août 1792, elle avait la charge d'écrire aux commissaires envoyés en mission ${ }^{14}$, et dès le 3 septembre de la même année, celle de rédiger le Bulletin national ${ }^{15}$. C'est ainsi qu'elle communiquait dans tout l'Empire les directives de l'Assemblée, ses décrets et les discours de ses députés. Pour être plus précise, la commission de correspondance était celle qui adressait les ordres de l'Assemblée aux commissaires envoyés en mission et celle qui recevait, en contrepartie et dans un premier temps, leurs nouvelles, parfois confidentielles étant donné le caractère particulier des informations concernant le maintien de l'ordre et de la tranquillité publique. C'était aussi la commission chargée de rédiger le Bulletin national qui devait rendre compte de la situation de l'Empire et donner les détails de la correspondance des 
commissaires envoyés en mission aux armées, pour combattre les calomnies qui accusaient l'Assemblée de ne pas communiquer les informations aux citoyens. C'est ainsi qu'Armand Guy Simon de Kersaint intervint à l'Assemblée le 3 septembre 1792 : « On vous accuse d'avoir des lumières que vous ne communiquez pas au public, allez au-devant de cette calomnie et faites un bulletin national $»^{16}$.

\section{Gains. Le Comité des pétitions et de correspondance de la Convention nationale}

Dès le premier jour de réunion, le 17 octobre 1792, les députés du Comité des pétitions et de correspondance poursuivirent les mêmes travaux que pendant les derniers temps de l'Assemblée législative ${ }^{17}$, avec cependant deux nouveautés importantes: ce comité s'occuperait de gérer l'intervention des pétitionnaires à la barre et au Bureau des renvois et des correspondances. À partir du mois de novembre 1792, les pétitionnaires qui voulaient lire personnellement au Corps législatif leurs vœux, leurs plaintes, leurs pétitions ou leurs demandes devaient se présenter devant le Comité des pétitions et de correspondance, et non plus devant le président de l'Assemblée ${ }^{18}$. En outre, le Bureau des renvois et des correspondances devint alors une section du Comité des pétitions et de correspondance qui n'était plus contrôlé par le président ni les secrétaires de l'Assemblée, comme cela se passait depuis $1789^{19}$. L'importance de ce bureau est due au fait qu'il agissait comme premier filtre de toute la correspondance qui arrivait au Corps législatif, et cela, parce qu'il était responsable, entre autres travaux, d'adresser aux députés et aux comités la correspondance qu'ils recevaient particulièrement, mais aussi d'ouvrir, enregistrer et renvoyer aux comités la correspondance adressée à l'Assemblée ou à son président et enfin de présenter à ce dernier les informations susceptibles d'être communiquées au Corps législatif ou d'être envoyées, en cas d'urgence, à certains comités.

Tableau $\mathrm{n}^{\circ} 1$

\begin{tabular}{|c|c|}
\hline Assemblée nationale constituante / Assemblée législative & Convention nationale \\
\hline Président & Comité des pétitions et de correspondances \\
\hline $\begin{array}{l}\text { Bureau des renvois et } \\
\text { des correspondances }\end{array}$ & $\begin{array}{l}\text { Bureau des renvois et } \\
\text { des correspondances }\end{array}$ \\
\hline
\end{tabular}

6 Sous la Convention nationale, et pendant quelques mois seulement, toute la correspondance qui arriva au Corps législatif passa par les mains du Comité des pétitions et de correspondance. Ce comité devint le premier filtre de la correspondance. À partir du mois d'octobre 1792, les citoyens pour se faire entendre de l'Assemblée devaient passer par ce nouveau filtre, et non plus par celui du président de l'Assemblée, comme c'était le cas sous les deux Assemblées précédentes. (Voir le tableau ํํำ) 


\section{Pertes. Le président de l'Assemblée}

7 Le Comité des pétitions et de correspondance prit sa forme finale et son importance considérable pendant le mois de novembre 1792 au moment où il vint à contrôler deux des trois compétences gérées jusqu'alors par le président de l'Assemblée. Depuis le début de l'Assemblée nationale constituante, le président avait entre les mains trois compétences très importantes : la direction des débats parlementaires, le contrôle des pétitionnaires et la direction du Bureau des renvois et des correspondances. Le président de l'Assemblée ouvrait et fermait les séances parlementaires, il préparait l'ordre du jour conjointement avec le comité central, il donnait, coupait et retirait la parole aux députés, posait les questions sur lesquelles ces derniers devaient délibérer et prendre position, refaisait le compte des voix après les votations, fermait les discussions et essayait de maintenir l'ordre dans la salle. Il choisissait aussi les pétitionnaires qui pouvaient se présenter à la barre et, comme il contrôlait le Bureau des renvois et des correspondances, il choisissait également les informations qui devaient être lues aux députés. Ces trois compétences, ensemble et même séparément, pouvaient permettre au président de l'Assemblée, à un moment déterminé, d'orienter le débat parlementaire dans un sens ou dans un autre, de favoriser ou non un point de vue ou une tendance politique. Aussi n'estil pas étonnant qu'il y ait eu d'importantes discussions sous l'Assemblée législative à propos des compétences du président ${ }^{20}$, et que Claude Basire ait rappelé le 17 novembre 1791 à Vincent Marie Viennot de Vaublanc, président ce jour-là, au milieu d'une vive discussion: "Vous êtes juge et partie $»^{21}$. Pas étonnante non plus la véritable lutte politique entre députés jacobins et feuillants pour obtenir le contrôle de la présidence le 23 juillet $1792^{22}$. Le rôle de filtre que le président de l'Assemblée exerçait sur les informations était bien connu de tous les députés, et même combattu pendant les premiers jours de l'Assemblée législative, quand une partie des députés du côté gauche avaient essayé d'éliminer tout filtrage des pétitionnaires et de la correspondance qui parvenait à l'Assemblée ${ }^{23}$. L'établissement du Comité des pétitions et de correspondance avec ses deux nouvelles compétences signifiait que le président était démis de deux prérogatives importantes, et qu'il ne lui en restait qu'une, celle de gérer le débat parlementaire. Autrement dit, cela signifiait que son influence sur les discussions dans le Corps législatif afin d'élaborer les lois avait été diminuée. (Voir le tableau nº 2)

Tableau $\mathrm{n}^{\circ} 2$

\begin{tabular}{|l|l|}
\hline Assemblée nationale constituante / Assemblée législative & Convention nationale \\
\hline & Président
\end{tabular}




\section{Centralisation. Le comité central d'Alba Lasource}

8 Le Comité des pétitions et de correspondance qui se forma mi-novembre fut en réalité l'aboutissement partiel d'une proposition faite à l'Assemblée le 10 octobre 1791 par DavidMarie Lasource, connu aussi sous le nom d'Alba Lasource ${ }^{24}$. Lasource proposa l'établissement d'un comité central chargé de recevoir toute la correspondance arrivant au Corps législatif et de la distribuer aux différents comités de l'Assemblée. Ce comité qui échappait au contrôle du président devait assurer, contrairement au Bureau des renvois et des correspondances, une série de tâches complémentaires, à savoir :

«Art. $1^{\mathrm{er}}$. Le comité général recevra toutes les pétitions, adresses, projets, mémoires, sur toutes sortes d'objets. Art. 2. Il prendra note sommaire de l'objet de chaque envoi qui lui sera fait, et l'inscrira sur un registre, en mettant un numéro à chaque pièce et en recommençant chaque mois, pour éviter la confusion. Art. 3. Cette première opération faite, il fera parvenir de suite chaque objet au comité qui devra en connaître. Art. 4. S'il y avait un certain nombre d'objets qui fût de la compétence d'un comité qui n'existe pas encore, le comité général en demandera la création. Art. 5. S'il arrivait que les pétitions, questions, etc., parvinssent à quelques comités sans être parvenues au comité central, avant toute opération ces comités seront tenus de présenter l'original desdites pétitions, etc., au comité central qui en prendra note. Art. 6 . Les comités ne pourront faire aucune réponse ni donner aucun éclaircissement, avis ou décision, sans en donner note au comité central. Art. 7. Les réponses et éclaircissements, avis et décisions seront inscrits à la page du registre opposée à la demande et correspondront avec elle par numéro. Art. 8. Les comités seront tenus de fournir dans un mois au plus tard leur note sur chaque numéro que le comité central leur aura fait parvenir. Le comité central les en sommera s'ils ne l'ont point fait, et s'il restait un trop grand nombre d'affaires en arrière. [...] Le comité central sommera au bout d'un mois le Comité de rendre compte à l'Assemblée. Si celui-ci ne le fait pas, le comité central instruira l'Assemblée nationale qui décidera sur son rapport. Le double des registres du comité sera déposé dans un des bureaux de l'Assemblée, où chaque membre pourra en prendre connaissance, toutes les fois que bon lui semblera, pendant 3 jours de la semaine. $»^{25}$

9 L'objectif poursuivi par Lasource avec cette proposition était d'éviter que la correspondance renvoyée aux comités ne fût pas communiquée à l'Assemblée, et que l'Assemblée fût à l'écart des décisions que les comités prenaient sur les informations qu'ils avaient reçues. C'était un objectif qui voulait à son tour restreindre l'indépendance des comités par rapport au pouvoir de l'Assemblée, bien connue de tous les députés des assemblées parlementaires et dénoncée par Georges-Auguste Couthon le $1^{\mathrm{er}}$ octobre 1791 :

«Des mémoires, des instructions, des adresses, des pétitions étaient directement adressés aux comités, on les prenait ou non en considération : cela dépendait d'un concours de correspondance qu'il est inutile d'approfondir ; mais alors l'Assemblée nationale, qui n'avait aucune connaissance des différents renseignements, prononçait sans connaître les différents moyens qui dictaient son décret. ${ }^{26}$

$10 \mathrm{Au}$ mois de novembre 1791, la proposition d'Alba Lasource ne fut pas acceptée et, en octobre 1792, la Convention nationale n'accorda pas au Comité des pétitions et de correspondance les tâches complémentaires que Lasource avait établies dans son projet pour l'établissement d'un comité central. Les comités de la Convention nationale ne se virent jamais dans l'obligation d'adresser au Comité des pétitions et de correspondance les informations qui avaient échappé à son contrôle et qu'ils avaient reçues par d'autres voies, pas davantage que lui communiquer les décisions qu'ils adoptaient concernant la correspondance reçue. Néanmoins, la proposition de Lasource aboutit partiellement au 
Comité des pétitions et de correspondance. Sous la Convention nationale, ce fut un comité et non le Bureau des renvois et des correspondances sous la direction du président de l'Assemblée qui fut chargé de recevoir et de distribuer, à partir de novembre 1792, toute la correspondance parvenant au Corps législatif.

\section{Porter. Flux d'informations sortantes, entrantes et internes}

11 Le Comité des pétitions et de correspondance fut important, surtout pendant les premiers mois de la jeune République, par le contrôle qu'il exerça sur des flux d'informations qui pourraient être regroupées en trois catégories : sortantes, entrantes et internes ${ }^{27}$. En ce qui concerne les flux d'informations de sortie, ceux qui transmettaient les informations de la Convention nationale à d'autres partenaires, ou bien des mandataires aux commettants, le Comité des pétitions et de correspondance, étant donné qu'il avait absorbé le contrôle du Bureau des renvois et des correspondances, eut la charge de rédiger les lettres au nom du président de l'Assemblée. Cette correspondance était normalement adressée aux ministres, aux directoires de département et de district, aux tribunaux, aux municipalités, aux gardes nationales, aux régiments et aux particuliers. En ce sens, le Comité des pétitions et de correspondance travailla à transmettre toutes les informations nécessaires pour la bonne continuation de leurs travaux. Par cet envoi de lettres, de discours et d'adresses, il communiquait les instructions de l'Assemblée, les procédures ainsi que les pratiques nouvelles qu'ils devaient adopter pour marcher à l'unisson avec le Corps législatif ${ }^{28}$. Ce comité fut aussi chargé de communiquer aux commissaires envoyés en mission, via les flux d'informations de sortie, les instructions de travail, les travaux à réaliser, les informations propres de l'Assemblée et les informations d'endoctrinement. Mise à part sa correspondance avec les commissaires envoyés en mission, le comité correspondait aussi avec les généraux. Finalement, son poids le plus important sur les flux d'informations sortantes se trouve dans la rédaction du Bulletin national. Six députés et huit secrétaires commis de ce comité, qui travaillaient tous les jours, avaient sous leur responsabilité la réalisation de ce bulletin qui diffusait les opérations de l'Assemblée, celles des commissaires envoyés en mission et quelques pétitions et adresses lues à la barre. En ce qui concerne les flux d'informations entrantes, celles qui étaient transmises de toutes parts de la République à la Convention nationale, des commettants aux mandataires, le Comité des pétitions et de correspondance dut assurer la gestion de toute la correspondance arrivant au Corps législatif à partir du 11 novembre $1792^{29}$. Toutes les lettres qui n'étaient pas adressées nominalement à un député ou à un comité en particulier étaient ouvertes et lues par le Comité des pétitions et correspondance qui décidait de leur sort final. En ce sens, ce comité exerçait une importante tâche de filtrage ; filtrage que les citoyens pouvaient contourner en adressant des lettres à un député ou à un comité en particulier. En outre, ce comité contrôlait aussi les informations provenant des commissaires envoyés en mission et des généraux. À tout moment, il était au courant de leurs opérations ainsi que des problèmes et difficultés de leurs missions. C'est à lui que revenait la décision de communiquer tout ou partie à l'Assemblée. En ce qui concerne les flux d'informations internes, ce comité avait une influence remarquable. La plupart des informations reçues par les comités et les commissions provenaient d'une décision adoptée par le Comité des pétitions et de correspondance. Seules les lettres qu'ils recevaient nominalement échappaient au 
contrôle de ce comité. Le Comité des pétitions et de correspondance ou, pour mieux dire, ses membres et ses secrétaires commis agirent en qualité de porters -concept décrit par Kurt Lewin- en ce sens que les porters sont ceux qui occupent des postes-clés pour faire circuler les informations. Comme le souligne Eduard Resbier: "[les porters] sont semblables à des relais qui peuvent se comporter comme des filtres des informations, en sélectionnant celles qu'ils considèrent comme les plus importantes. Cela pose cependant un problème d'altération des communications dans la mesure où le choix mentionné est conditionné par les valeurs, les stratégies, les buts et, en définitive, par tout ce que le porter considère important $»^{30}$.

\section{Vérité/Vérités. Le Bureau de l'esprit public et la commission de correspondance sous l'Assemblée législative et la Convention nationale}

12 À partir du 10 août 1792, la Commission extraordinaire des douze, qui travaillait aux côtés du Comité des pétitions depuis sa mise en place, commença à encourager une politique de diffusion des nouvelles pour combattre les calomnies et les diatribes que, selon leurs rapports, les ennemis de la patrie diffusaient. La Commission extraordinaire des douze, créée le 17 juin 1792 pour sauver la Constitution, la liberté et l'Empire ${ }^{31}$, était d'avis qu'il fallait imprimer des "vérités", tout comme de son côté la Cour avait payé différents écrivains pour corrompre l'opinion publique.

«Messieurs, vous avez déjà été à même de voir, par les rapports de vos commissaires chargés d'assister à l'inventaire des papiers de la liste civile, que la cour soudoyait une quantité énorme d'écrivains pour corrompre l'opinion publique. Plus on a imprimé de calomnies et de diatribes, plus vous devez maintenant imprimer de vérités $»^{32}$.

13 Pendant la journée du 10 août 1792, on avait remarqué que les désordres avaient pris fin quand l'Assemblée avait communiqué au peuple ses décrets ${ }^{33}$. Le peuple français y était invité à former une Convention nationale, et le roi restait suspendu de façon provisoire jusqu'au moment où cette nouvelle assemblée prononcerait les mesures à prendre pour assurer la souveraineté du peuple et le règne de la liberté et de l'égalités ${ }^{34}$. Informer des décrets adoptés par l'Assemblée et diffuser «la vérité »/« les vérités » étaient devenus une priorité pour les députés qui le 10 août 1792 prêtèrent le serment de maintenir la liberté et l'égalité ou de mourir à leur poste ${ }^{35}$. À cet égard, douze d'entre eux furent envoyés dans les quatre armées, celle de l'Ouest, celle du Rhin, celle du Midi et celle du Centre, pour relater les événements qui avaient eu lieu à Paris et les causes qui les avaient produits $^{36}$. Une fois le roi suspendu provisoirement, et les six ministères renouvelés Intérieur, Guerre, Contributions publiques, Justice, Marine et Affaires étrangères-, la politique de diffusion des informations que la Commission extraordinaire des douze mit sur pied vint s'articuler rapidement en deux pôles: l'un entre les mains du pouvoir exécutif, et l'autre entre celles du pouvoir législatif. Plus explicitement, l'un entre les mains du ministre de l'Intérieur, Jean-Marie Roland de la Platerie, l'autre entre celles de la Commission extraordinaire des douze qui était chargée, depuis sa composition, de penser et de proposer à l'Assemblée les mesures les plus convenables pour le maintien de l'ordre et de la tranquillité publique ${ }^{37}$. Le 18 août 1792, afin de combattre les discours contre-révolutionnaires, la Commission extraordinaire des douze demanda de mettre à la disposition du ministère de l'Intérieur 100000 livres pour imprimer et distribuer, dans les 
départements et les armées, tous les écrits qui pouvaient éclairer les esprits sur les trames criminelles des ennemis de l'État et sur les vrais maux qui déchiraient la patrie ${ }^{38}$. Le Bureau de l'esprit public créé à la fin du mois de mars $1792^{39}$, et sous la direction de Roland, fut chargé de cette distribution.

Le 3 septembre 1792, dans cette même ligne, Kersaint, qui avait été envoyé en mission à l'armée du Centre pour informer sur les faits arrivés le 10 août 1792, demanda la rédaction d'un bulletin national pour arrêter les désordres. Selon ce député, les désordres avaient lieu parce que le peuple méconnaissait «la vérité » et que les ennemis le trompaient facilement. La commission de correspondance, sous les ordres de la Commission extraordinaire des douze, fut chargée de cette rédaction. Le discours de Kersaint fut le suivant :

«Messieurs, les désordres publics sont les fruits de l'erreur et vous savez avec quelle avidité vos ennemis ont saisi ce moyen pour égarer le peuple. C'est à vous qu'il appartient de lui faire connaître la vérité. En cet instant peut-être on jette dans le public des nouvelles exagérées, sur lesquelles il faudra revenir, et l'on espère par ce moyen arrêter l'ardeur civique de Paris, et en cas de revers, car on peut en éprouver, jeter le découragement dans les âmes trompées par une fausse espérance. Il faut donc faire connaître au peuple la vérité ; il faut lui faire parvenir les faits dans leur exactitude et vos décrets dans leur intégrité. On a fait autrefois un logographe contre-révolutionnaire, il faut avoir un logographe national. Il serait possible de rappeler quelques-uns des citoyens qui se livraient à ce travail avec tant d'intelligence et qui sont patriotes. Il faut que vous établissiez près de vous des écrivains qui répandront les nouvelles, les faits et vos opérations d'une manière certaine et légale. Des journalistes bien intentionnés, mais mal placés ici, les impriment souvent d'une manière inexacte. $»^{40}$

Du moment où ces mesures furent acceptées, le pouvoir exécutif et le pouvoir législatif commencèrent à travailler pour communiquer les décrets de l'Assemblée, "la vérité ", "les vérités ». À partir de là se mirent en relation tout un ensemble de bureaux et d'institutions pour accomplir cet objectif. Plus précisément, c'est par l'intermédiaire du ministre de l'Intérieur - dans le cadre du pouvoir exécutif- et de la Commission extraordinaire des douze -dans le cadre du pouvoir législatif- que les organes de diffusion -le Bureau de l'esprit public et la commission de correspondance- furent mis en contact avec, d'une part, les bureaux qui recevaient la correspondance des départements et, d'autre part, les agents et les commissaires envoyés en mission. (Voir tableau $\mathrm{n}^{\circ}$ 3) Le ministre de l'Intérieur établit une liaison entre le Bureau de l'esprit public et le bureau qui recevait la correspondance des départements ${ }^{41}$. Et, en relation avec ces deux bureaux, il dirigea les opérations des agents envoyés en mission par le conseil exécutif provisoire pour propager les écrits votés par l'Assemblée et communiquer les lois adoptées afin de protéger l'État et combattre les ennemis.

«Le Ministre de l'Intérieur donne au citoyen............ la mission de parcourir les départements d.................... d'y répandre les écrits dont l'Assemblée nationale a voté l'impression et l'envoi dans les départements ; de fixer l'attention des citoyens sur les lois que l'Assemblée nationale a décrétées et décrète journellement pour pourvoir à la sûreté de l'État contre l'invasion de ses ennemis, d'en presser partout l'exécution, de la recommander à la surveillance des bons citoyens; de les exciter à fabriquer des armes de toute espèce, à transformer en moyens de défense les métaux et tout ce qui peut y servir; de répandre de toutes parts les lumières et l'esprit public; de faire en un mot tout ce que pourra lui inspirer le plus ardent amour de la patrie, en se bornant néanmoins à ce que tout citoyen a individuellement le droit d'entreprendre; et je le recommande à ce titre, en tant que de besoin, aux bons offices des corps administratifs et des amis de la Patrie. ${ }^{42}$ 
côté, la Commission extraordinaire des douze qui travaillait étroitement avec le Comité des pétitions - l'organe des corps administratifs et des citoyens de l'Empire, selon les mots de Gossuin - et qui recevait depuis le 17 juin 1792 la correspondance concernant l'administration et la police - celle qui rendait compte des désordres- eut sous ses ordres la commission de correspondance qui, d'un côté, dictait les directives de l'Assemblée aux commissaires envoyés en mission et, de l'autre, rédigeait le Bulletin national.

Tableau $\mathrm{n}^{\circ} 3$
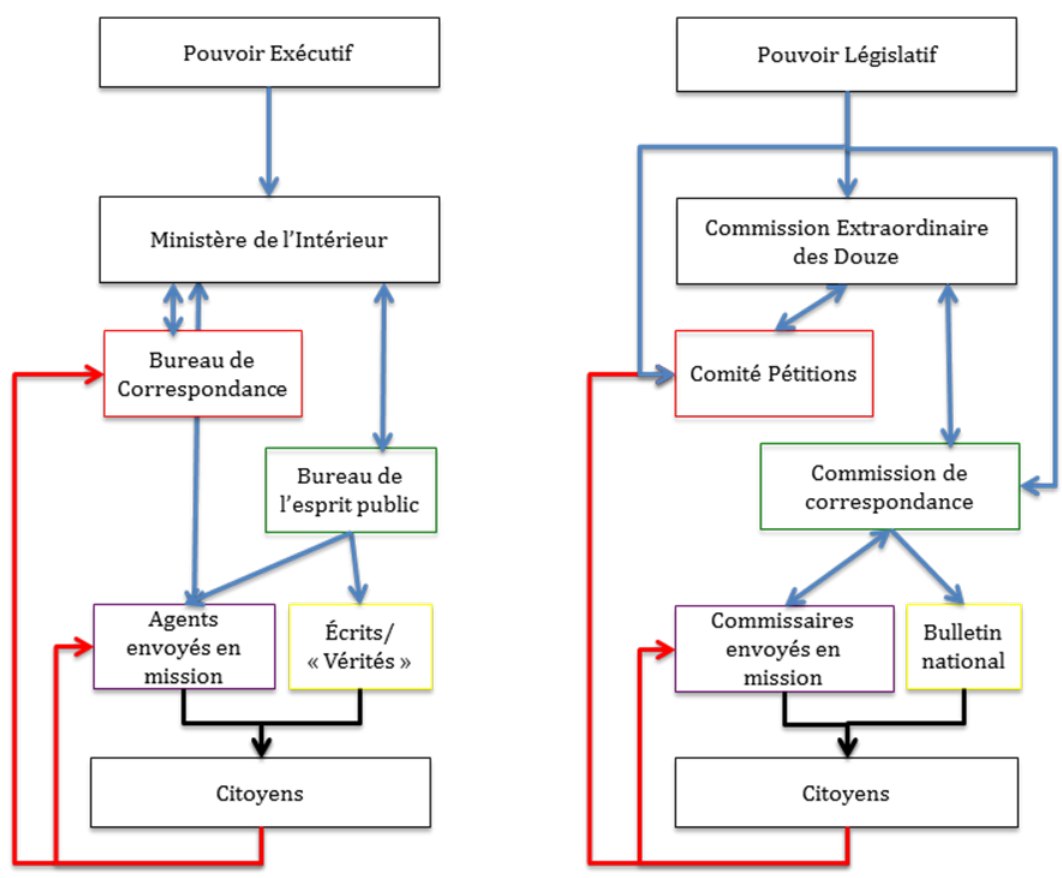

\begin{tabular}{|l|l|l|l|l|l|}
$\longrightarrow$ & $\begin{array}{l}\text { Contacts entre } \\
\text { organismes }\end{array}$ & $\longrightarrow$ & $\begin{array}{l}\text { Flux d'informations } \\
\text { sortantes }\end{array}$ & $\longrightarrow$ & $\begin{array}{l}\text { Flux d'informations } \\
\text { entrantes }\end{array}$ \\
\hline
\end{tabular}

Pour le maintien de l'ordre et de la tranquillité publique, pour la diffusion des décrets de l'Assemblée et «de la vérité »/ «des vérités », il paraissait nécessaire de mettre en relation, depuis le pouvoir exécutif et le pouvoir législatif, une combinaison de flux d'informations entrantes et sortantes, combinaison que la Convention nationale ne modifia pas complètement pour continuer à transmettre et recevoir des informations avec efficacité. Après le 22 septembre 1792, le Bureau de l'esprit public continua ses travaux sous la direction de Roland et la commission de correspondance resta attachée au Comité des pétitions, étant donné que la jeune République ne pouvait maintenir, face au Ministère de l'Intérieur qui jouissait de la confiance du pouvoir législatif et face au principe de la séparation des pouvoirs, une Commission extraordinaire des douze chargée de penser les mesures pour le maintien de l'ordre et de la tranquillité publique. Les commissions créées pareillement à la Commission extraordinaire des douze (17 juin 1792) ou à la Commission des douze (6 mars 1792), avaient leur sens uniquement quand le pouvoir législatif se méfiait du pouvoir exécutif et de sa force, et que ce premier décidait de prendre les rênes de la situation intérieure et extérieure du pays pour impulser et dynamiser les travaux de ce dernier ${ }^{43}$. 


\section{Méfiance. Le Comité de défense générale}

18 Le premier janvier 1793, Kersaint, qui avait demandé au mois de septembre 1792 la rédaction d'un bulletin national, demanda la création d'un Comité de défense générale pour aider les ministres dans la prise de décisions ${ }^{44}$. Quatre mois après le 10 août 1792 , le pouvoir exécutif ne jouissait pas d'une totale confiance de l'Assemblée. Ses dissensions internes montraient sa faiblesse, alors Kersaint proposa l'établissement d'une nouvelle Commission extraordinaire des douze pour impulser ses travaux. En quelques mois seulement, la méfiance envers le conseil exécutif provisoire, et plus concrètement envers le Ministère de l'Intérieur, de la part d'un secteur de l'Assemblée s'était tellement développée que même Maximilien-François-Isidore Robespierre avait demandé de retirer des mains de Roland le fonds de 100000 livres accordé le 18 août 1792 pour propager les décrets et décisions de l'Assemblée et «les vérités » censées combattre les écrits contrerévolutionnaires ${ }^{45}$. Or, ces fonds avaient permis, d'août 1792 à janvier 1793, de dynamiser le Bureau de l'esprit public.

Le $1^{\text {er }}$ janvier 1793, Kersaint qui avait été membre de l'Assemblée législative et connaissait bien le fonctionnement des commissions créées pour impulser les travaux du pouvoir exécutif, proposa à la Convention nationale la création d'un comité, pareil à la Commission des douze créée le 6 mars 1792, à partir de membres des comités suivants : pétitions, surveillance, agriculture, commerce, militaire et législation. Le Comité de défense générale demandé par Kersaint devait être composé de membres des comités suivants: guerre, finances, colonies, commerce, marine, diplomatie et constitution. Vingt-et-un membres au lieu de douze pour s'occuper, conjointement avec les ministres, des campagnes militaires et des affaires du moment, et renverser l'image que l'Angleterre pouvait avoir d'une France divisée par ses luttes internes et les profonds désaccords entre ses divers partis ${ }^{46}$. Malgré des similitudes en termes d'organisation avec la Commission des douze, le Comité de défense générale présentait une nouveauté importante : le Comité des pétitions et de correspondance, successeur du Comité des pétitions, et le Comité de sûreté générale, successeur du Comité de surveillance, n'en faisaient pas partie. Les travaux du Comité de sûreté générale n'avaient pas changé beaucoup par rapport aux travaux du Comité de surveillance, mais le Comité des pétitions et de correspondance avait vraiment changé. En effet, sous la Convention nationale il avait perdu une de ses compétences caractéristiques: la gestion du maintien de l'ordre et de la tranquillité publique. Ainsi, il ne s'occupa jamais de penser les mesures qui devaient être prises par le Corps législatif pour mettre un terme aux désordres, à la différence du Comité des pétitions entre octobre 1791 et mars 1792, et du Comité des rapports sous l'Assemblée nationale constituante, ses deux prédécesseurs. Ces deux comités, chacun en son temps, avaient reçu la correspondance concernant l'administration et la police, et ils avaient décidé, tout seuls ou bien avec l'accord de l'Assemblée, selon la gravité des désordres, de la meilleure façon de rétablir l'ordre et la tranquillité publique. Ces deux comités avaient travaillé côte à côte avec le comité chargé de localiser les auteurs des désordres, formant, sous chaque Assemblée parlementaire, un tandem parfait pour le maintien de l'ordre et de la tranquillité publique. Un tandem qui était formé sous l'Assemblée nationale constituante par le Comité des rapports et le Comité de recherches, et sous l'Assemblée législative, et jusqu'en mars 1792, par le Comité des pétitions et le Comité de surveillance ${ }^{47}$. Sous la Convention nationale, le Comité des pétitions et de correspondance devint 
d'une certaine façon le comité central que Lasource avait projeté en octobre 1791, c'est-àdire un important porter pour les informations entre commettants et mandataires et vice versa. Son absence dans la composition du Comité de défense générale au moment où il fut créé, le $1^{\mathrm{er}}$ janvier 1793, mit en évidence la métamorphose la plus importante que ce comité expérimenta pendant les premiers mois de la jeune République.

\section{Parallélismes. Le Comité de défense générale et la Commission extraordinaire des douze}

Comme la Convention nationale ne mit sur pied jusqu'à janvier 1793 aucune commission pareille à celle des douze, le Comité des pétitions et de correspondance s'occupa tout seul d'écrire aux commissaires envoyés en mission. Néanmoins, quand la Convention nationale institua le Comité de défense générale, et comme le Comité des pétitions et de correspondance ne fut pas appelé pour y être présent, les membres de ce comité décidèrent de s'y rattacher. Ils voulaient communiquer avec efficacité aux commissaires envoyés en mission les consignes adoptées dans le cadre de ce nouveau comité.

«Le président ayant ouvert la séance après la lecture du procès-verbal de la dernière séance, un membre a observé qu'il paraissait convenable de proposer à la Convention nationale de décréter que trois membres, pris dans le Comité de correspondance fussent autorisés à assister au Comité de défense générale pour s'y pénétrer des objets dont il était du devoir du Comité de correspondance d'instruire les commissaires de la Convention nationale, sur quoy les membres présents au comité ont chargé le citoyen Roux, l'un desdits membres, d'en faire demain la proposition à la Convention nationale au nom du dit comité. $\rangle^{48}$

Le 31 janvier 1793, immédiatement après que Louis-Félix Roux eut fait sa proposition au nom du Comité des pétitions et de correspondance, l'Assemblée l'accepta ${ }^{49}$. Le lendemain, trois membres de la section de correspondance du comité se réunirent au Comité de défense générale ${ }^{50}$. Prise entre les discussions existantes et la nécessité de communiquer un seul discours, la Convention nationale accepta de reprendre le même fonctionnement que sous l'Assemblée législative, où la Commission extraordinaire des douze dictait ses consignes à la commission de correspondance pour qu'elles soient communiquées aux commissaires envoyés en mission. Ces commissaires, au-delà de leurs travaux dans les départements frontaliers et les armées, s'occupaient de transmettre des informations dans les deux sens, ainsi que les décrets et les discours de l'Assemblée, afin d'unir dans un projet commun les commettants et les mandataires, projet commun que Robespierre définit de la façon suivante :

«Il faut une volonté une. Il faut qu'elle soit républicaine ou royaliste. Pour qu'elle soit républicaine, il faut des ministres républicains, un gouvernement républicain.

(...) Il faut que le peuple s'allie à la Convention et que la Convention se serve du peuple. $»^{51}$

Tant que le Comité de défense générale exista, le Comité des pétitions et de correspondance y eut toujours trois membres qui alternaient tous les quinze jours. Néanmoins, quand le Comité de salut public fut créé, tout changea. Le Comité de défense générale fut remplacé et le Comité des pétitions et de correspondances subit sa deuxième métamorphose. 


\section{Métamorphose. Le Comité de salut public et la Commission des dépêches}

23 La création du Comité de salut public le 6 avril $1793^{52}$ déclencha la deuxième métamorphose du Comité des pétitions et de correspondance qui, le lendemain même, perdit la compétence de correspondre avec les représentants en mission et trois jours plus tard, la gestion de la correspondance qui arrivait au Corps législatif. Le 7 avril 1793, le Comité de salut public décida que lui-même s'occuperait de correspondre avec les représentants en mission ${ }^{53}$ et, le lendemain, la Convention nationale décida de créer une commission pour examiner les lettres des ministres, des généraux et des représentants en mission. Cette commission était habilitée à décider des lettres qu'elle communiquerait au Corps législatif -intégralement ou résumées- et de celles qu'elle enverrait directement au Comité de salut public pour éviter les inconvénients possibles lors d'une lecture à l'Assemblée. La mise sur pied de cette commission signifia pour le Comité des pétitions et de correspondances la perte de son contrôle sur le Bureau des renvois et des correspondances. Le 10 avril 1793, cette commission reçut la charge de gérer toute la correspondance parvenant à la Convention nationale, et de renvoyer aux comités celle qui ne pouvait pas être gérée directement par le Corps législatif sans un rapport préliminaire $^{54}$. Le Bureau des renvois et des correspondances, qui était passé des mains du président aux mains du Comité des pétitions et de correspondance, passa dans les premiers jours d'avril 1793 aux mains de la Commission des dépêches. De la même manière la gestion des dons patriotiques passa aux mains de cette commission $^{55}$, compétence que le Comité des pétitions et de correspondance avait eue depuis le mois de novembre 1792. À la mi-avril 1793, le Comité des pétitions et de correspondance vit ses compétences réduites à deux : la gestion des pétitions qui n'étaient pas d'intérêt général et les demandes particulières ${ }^{56}$, mais aussi la rédaction du Bulletin national qui était envoyé chaque jour, à cette époque-là, aux armées de terre et de mer par l'intermédiaire des ministres de la Guerre et de la Marine, et aux départements, aux districts, aux établissements scolaires et aux sociétés patriotiques par l'intermédiaire du ministre de l'Intérieur. La mise sur pied d'une nouvelle Commission extraordinaire des douze, appelée cette fois-ci Comité de salut public, impliqua de réorganiser les flux d'informations, pour la première fois dans le cadre de la Convention nationale. L'établissement du Comité de défense générale ne les avait pas altérés, et ce comité avait agi pareillement à la Commission extraordinaire des douze de l'Assemblée législative. Néanmoins, le Comité de salut public agit de la même manière que le Comité des rapports de l'Assemblée nationale constituante, son antécédent - ainsi que du Comité des pétitions et de correspondance-, et il contrôla lui-même la correspondance des représentants en mission dans les départements et les armées. Gérer les flux d'informations à l'entrée et à la sortie n'était nullement une question mineure. Au moment où le Comité de salut public fut mis sur pied pour surveiller et accélérer l'action de l'administration confiée au conseil exécutif provisoire, devenant par ses compétences un comité fort exceptionnel, le contrôle de ces flux d'informations fut modifié et mis au service de ce comité.

$\mathrm{Au}$ moment où le contrôle des flux d'informations changea de mains, un organisme du Corps législatif s'affaiblit au détriment d'un autre qui vit grandir son influence sur le débat parlementaire. En avril 1793, le Comité de salut public vit grandir son influence au 
détriment du Comité des pétitions et de correspondance, qui avait accru son influence, en son temps, au détriment du président de l'Assemblée.

\section{Stratégies. Des luttes pour le contrôle de certaines compétences}

Depuis le mois d'octobre 1792, les députés de la Plaine et les girondins avaient un poids important sur les organismes qui géraient la correspondance, les flux d'informations entre les mandataires et les commettants. Ils étaient majoritaires dans le Comité des pétitions et de correspondance ${ }^{57}$; Roland, leader girondin, avait entre ses mains le ministère de l'Intérieur et le Bureau de l'esprit public, et les agents du conseil exécutif provisoire sous leurs ordres; et quand Kersaint demanda l'établissement du Comité de défense générale depuis les rangs girondins, ceux-ci eurent pour quelque temps une présence importante dans ce comités ${ }^{8}$. Pendant les premiers mois de la jeune République, ceux qui s'occupaient de propager «la vérité »/ "les vérités", de communiquer les consignes de l'Assemblée aux commissaires de la Convention et aux agents du conseil exécutif provisoire envoyés en mission, ceux qui rédigeaient le Bulletin national, ceux qui décidaient des pétitions et des pétitionnaires qui devaient être écoutés par le Corps législatif, étaient majoritairement des individus appartenant aux rangs des girondins et de la Plaine. La création du Comité de salut public et de la Commission des dépêches signifia la fin de ce contrôle sur les flux d'informations ainsi que de cette influence sur le débat parlementaire et les mandataires. Le Comité de salut public, qui remplaça le Comité de défense générale et prit le contrôle de la correspondance des représentants en mission et des informations les plus délicates arrivant à la Convention nationale, était composé d'un député de la Plaine et de huit députés montagnards ${ }^{59}$, tandis que la Commission des dépêches, qui absorba le Bureau des renvois et des correspondances, était composée d'un député de la Plaine, d'un député girondin et de trois députés montagnards ${ }^{60}$. La mise sur pied du Comité de salut public et de la Commission des dépêches dans les premiers jours d'avril 1793 permit aux députés montagnards de renforcer leur contrôle sur des compétences importantes. Des compétences qui, si elles n'avaient pas été transférées à ces deux nouveaux organismes -le Comité de salut public et la Commission des dépêches -, seraient restées aux mains des deux comités -le Comité de défense générale et le Comité des pétitions et de correspondance- dont ils n'avaient pas le contrôle et que la Convention nationale ne devait pas renouveler à ce moment-là. Quelques jours avant le 6 avril, le Comité de défense générale et le Comité des pétitions et de correspondance avaient été renouvelés et la présence des montagnards n'avait pas été capable de soustraire ces deux comités à l'influence des députés de la Plaine et des girondins ${ }^{61}$. Néanmoins, la présence majoritaire de députés montagnards dans les organismes qui géraient la correspondance en avril 1793 ne fut pas obtenue d'un jour à l'autre. Les députés montagnards avaient augmenté progressivement leur présence réelle dans le Comité des pétitions et de correspondance ${ }^{62}$, et à la fin du mois de mars ils avaient réussi à diminuer l'influence des députés girondins et de la Plaine dans le Comité de défense générale. Le dernier renouvellement de ce comité, le 26 mars 1793, leur avait permis d'y obtenir une présence non négligeable ${ }^{63}$; présence qui les motiva peut-être à appuyer l'établissement d'une nouvelle Commission extraordinaire des douze -le Comité de salut public- au lieu de proposer une réadaptation du Comité de défense générale aux nouveaux besoins de la Convention nationale ${ }^{64}$. Dans le cadre des assemblées 
parlementaires, la réadaptation d'un comité ne signifie pas automatiquement son renouvellement, tandis que la mise sur pied d'un nouveau comité ouvre les portes à de nouvelles élections; nouvelles élections qui devaient permettre, dans le cas du Comité de salut public, de dépasser les compositions où l'affrontement constant entre les membres ne favorisait pas l'adoption rapide de décisions ${ }^{65}$. En plus, le fait de décréter que le Comité de salut public devait être élu par appel nominal, et non pas à partir d'une liste élaborée par le Bureau de la présidence, comme cela avait été le cas lors du renouvellement du Comité de défense générale le 26 mars, devait favoriser un changement dans la composition de ce nouveau comité. Dans le cadre des assemblées parlementaires, la création d'un nouveau comité ou d'une nouvelle commission pour mener à bien des tâches qu'un autre comité ou une autre commission assumait déjà, pouvait répondre à une stratégie visant à transférer certaines compétences des mains de certains individus ayant une tendance politique $\mathrm{X}$ - aux mains de certains autres -ayant une tendance politique Y. Sinon, pourquoi créer une Commission des dépêches alors qu'il y avait un Comité des pétitions et de correspondance? Pourquoi créer un Comité de salut public alors qu'existait le Comité de défense générale ? Celui-ci ne pouvait-il pas être adapté ? Et pourquoi ne pas réduire de vingt-cinq à neuf le nombre de membres du Comité de défense générale sans procéder à de nouvelles élections? Cette pratique avait été employée auparavant, pour citer un exemple parmi d'autres, le 5 décembre 1791 lorsque les Comités de la dette publique et de la caisse extraordinaire, de la trésorerie nationale, de la dépense publique et des contributions publiques se réduisirent eux-mêmes par la voie du scrutin $^{66}$.

\section{Absences. Les députés du Comité des pétitions et de correspondance}

26 Le 2 octobre 1792, la Convention nationale décida que le Comité des pétitions et de correspondance aurait vingt-quatre membres, après avoir entendu le rapport de Gossuin concernant les comités ${ }^{67}$. Trois semaines plus tard, elle décida que les comités seraient renouvelés par moitié tous les deux mois, et que le sort déciderait des membres qui devraient rester dans le cadre du comité et de ceux qui devraient partir. La Convention nationale venait d'accepter ce jour-là une proposition de Gossuin sur le renouvellement des comités, présentée par ce député le 25 octobre $1792^{68}$. Le Comité des pétitions et de correspondance, à la demande de son président, devait avoir vingt-quatre membres et être renouvelé tous les deux mois. Mais il ne put jamais compter sur la présence de vingtquatre membres pour mener à bien ses travaux, et le 25 novembre 1792 Gossuin fit la proposition suivante à la Convention nationale : «Je demande qu'il soit rendu compte, chaque quinzaine, des membres qui n'assisteront pas aux séances de leurs comités. " ${ }^{69} \mathrm{~A}$ la date du 25 novembre 1792, le Comité des pétitions et de correspondance avait été réuni treize fois, et seulement pour une journée, le total des membres présents s'élevait à quinze. Généralement, les réunions comptaient entre huit et dix membres ${ }^{70}$. La Convention nationale accepta immédiatement la proposition de Gossuin afin d'éviter des absences réitérées mais l'acceptation de cette proposition ne renversa pas la dynamique. La situation du Comité des pétitions et de correspondance n'était pas isolée et exceptionnelle. La plupart des comités manifestèrent à la fin décembre 1792 leur regret de constater les absences de leurs membres. À cette époque-là, on devait les renouveler et des discussions eurent lieu pour décider comment et quand ils devaient l'être. 
«Cambacérès. Le Comité de législation est composé de 48 membres, nous n'avons jamais été plus de 18. La proposition du citoyen Carpentier est juste : il conviendrait que les comités fissent imprimer la liste de ceux des membres qui ne se rendent pas exactement à leur poste, et que ces derniers soient exclus pour être punis de leur négligence.»

«Treilhard. Hier, au Comité de liquidation, nous avons voulu procéder au renouvellement et le sort a décidé que ceux qui n'y avaient jamais paru resteraient dans le comité. Nous avons déchiré la liste. $»^{71}$ 1792 la mesure suivante : les députés absents seraient inclus dans la liste des députés qui devraient abandonner le comité ${ }^{72}$. Le Comité des pétitions et de correspondance obéit immédiatement à la mesure adoptée par la Convention nationale et, le soir même, il enregistra dans ses procès-verbaux les noms des députés qui devaient abandonner le comité à cause de leur absence ${ }^{73}$. Dans les premiers jours de janvier 1793, eut lieu le premier renouvellement des comités, malgré les réticences de certains députés à les renouveler avant le jugement du roi $^{74}$. Le Comité des pétitions et de correspondance fut renouvelé le 5 janvier 1793. Douze membres furent appelés à le composer à côté des douze qui y restaient ${ }^{75}$. Néanmoins, le renouvellement ne renversa pas la situation des absences réitérées et la coordination des trois sections qui le composaient resta aux mains d'une dizaine de membres qui assistaient plus ou moins régulièrement à leurs réunions. Les renouvellements postérieurs du Comité des pétitions et de correspondance ne renversèrent pas la situation non plus; pas davantage que l'intervention le 8 brumaire an II de Gossuin, à cette époque-là membre du Comité de la guerre, pour dénoncer à nouveau à la Convention nationale les absences des députés lors des travaux des comités. Ce jour-là, aucun comité ne présenta ses travaux devant la Convention nationale et Gossuin prit la parole pour demander au Comité de salut public de remplacer les individus qui pendant huit jours de suite n'assisteraient pas aux comités.

«Depuis quelques jours, je m'aperçois que les comités ne tiennent pas leurs séances. Ce n'est que par notre assiduité à nos devoirs que nous pouvons sauver la patrie. Nous sommes 20 membres au Comité de la guerre. Depuis un mois, sept ou huit seulement assistent aux séances. Il est vrai qu'il y en a beaucoup en commission. Je demande que tout membre qui sera absent huit jours de suite soit remplacé, sur la présentation du Comité de salut public. $»^{76}$

La Convention nationale accepta la proposition de Gossuin ainsi que celle de PhilippeAntoine Merlin de Douai de réduire de huit à trois les jours d'absence et de communiquer le nom des absents au département qui les avait élus députés.

«Il vaudrait mieux, à mon avis, décréter que tout membre d'un comité qui aura manqué trois jours de suite à ses séances, sera dénoncé, à la Convention nationale, et que son nom sera envoyé à son département. $»^{77}$

Malgré l'adoption de ces mesures, le Comité des pétitions et de correspondance continua à souffrir des absences de ses membres. Sa réorganisation au mois d'août 1794, réduisant le nombre de ses membres de vingt-quatre à douze, ne renversa pas la situation ${ }^{78}$. Parmi les 128 députés qui furent nommés membres ou suppléants entre le mois d'octobre 1792 et le mois d'octobre 1795, seulement 54 assistèrent à un moment ou à un autre aux réunions de ce comité, c'est-à-dire, 42,18 \% du total des membres appelés à le composer ${ }^{79}$. Les réunions du Comité des pétitions et de correspondance devaient avoir lieu tous les mercredis et tous les samedis ${ }^{80}$, mais les députés de ce comité ne se réunissaient pas toujours, et plusieurs fois ils changèrent le jour de la réunion. Pendant les premiers mois, les réunions se succédèrent régulièrement, mais d'octobre 1793 à septembre 1794 elles 
eurent lieu plus épisodiquement, exception faite des mois de germinal et messidor an II. La réorganisation de ce comité en fructidor an II réactiva pendant quelques mois la fréquence des réunions. Les députés étaient alors appelés à se réunir tous les quartidis et tous les nonidis à midi ${ }^{81}$. Durant un certain temps, la mesure fut suivie mais, à partir de prairial an III et jusqu'à la dissolution du comité, les députés se retrouvèrent seulement une fois par mois ${ }^{82}$. (Voir le graphique $\mathrm{n}^{\mathrm{o}} 1$ )

Graphique $n^{\circ} 1$

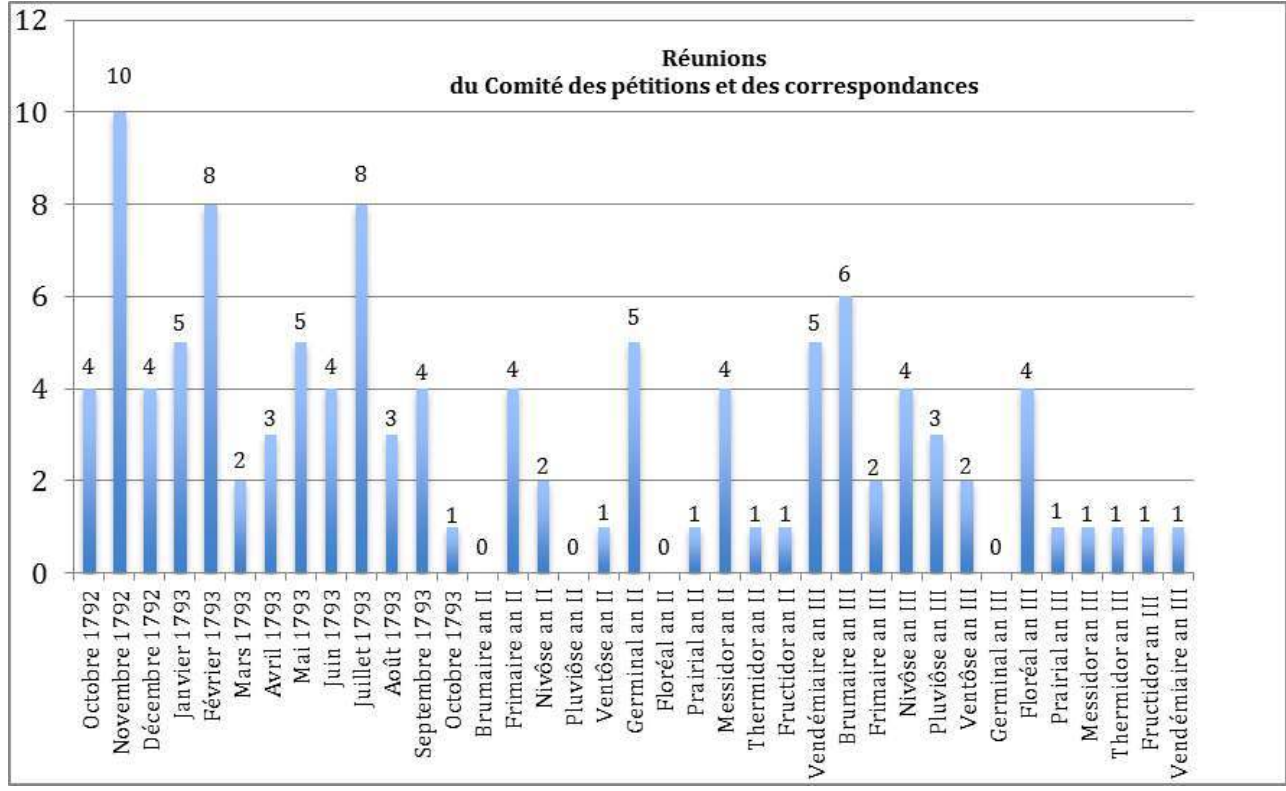

Peut-on penser que les travaux du Comité des pétitions et de correspondance n'aient pas eu besoin d'un nombre important de réunions? Le comité n'avait pas l'habitude de présenter des rapports à la Convention nationale, exception faite de ceux qu'il présenta pour rendre compte de la voix des commettants entre octobre 1792 et mars $1793^{83}$. D'ailleurs, ses procès-verbaux précisent seulement les mouvements du personnel dans le cadre de ce comité et ses mesures pour distribuer efficacement le Bulletin national. En fait, le travail des membres du comité consistait à surveiller le bon fonctionnement de ses trois sections : pétitions, correspondance, renvois et dons patriotiques. Trois sections qui se réduisirent à deux au moment où la Commission des dépêches absorba le Bureau des renvois et des correspondances entre le 8 et le 10 avril 1793 ; trois sections, ou deux en fonction du moment, organisées hiérarchiquement -chefs, sous-chefs, commis et garçons de bureau-, qui menèrent à terme les travaux qui leur étaient confiés.

\section{Continuité. Les secrétaires commis du Comité des pétitions et de correspondance}

31 Le Comité des pétitions et de correspondance n'eut jamais les mêmes compétences d'octobre 1792 à octobre 1795. Il ne fut jamais dominé par les mêmes députés ni par la même tendance politique. Ses compétences étaient augmentées ou diminuées et sa trajectoire n'était pas la même que celle de ses prédécesseurs. Il avait perdu la mission de gérer les désordres qui avaient lieu en France, et il ne figurait plus à côté du comité chargé de localiser les auteurs des troubles, formant avec ce dernier un tandem parfait 
pour le maintien de l'ordre et de la tranquillité publique. Il n'était plus le Comité des pétitions de l'Assemblée législative qui travaillait à côté du Comité de surveillance, pas davantage que celui des rapports qui travaillait à côté du Comité des recherches, et qui était devenu sous la Convention nationale l'antécédent du Comité de salut public. Néanmoins, le Comité des pétitions et de correspondance eut jusqu'à la fin de ses jours cinq des six secrétaires-commis de son prédécesseur, le Comité des rapports de l'Assemblée nationale constituante. Ainsi Garnier, Hussennet, Chaulay, Dupuis et Vaillant qui avaient travaillé sous les ordres du Comité de rapports depuis l'été 1789 étaient encore présents le 9 prairial an III, jour au cours duquel ils furent félicités pour avoir combattu le mouvement révolutionnaire qui venait d'éclater, en prenant les armes aux ordres du comitér ${ }^{84}$. Les membres et les suppléants du comité pouvaient changer tous les deux ou trois mois, et rentrer au comité quelques semaines plus tard s'ils étaient à nouveau élus, mais les individus qui lisaient les vœux, les plaintes, les pétitions et les demandes des commettants étaient les mêmes depuis juillet 1789, avec une différence importante cependant puisqu'ils avaient perdu le contrôle d'une compétence : la lecture de la correspondance qui donnait le détail des désordres qui avaient lieu en France. Or, jusqu'au mois de septembre 1792, ils avaient géré cette compétence. Garnier, Hussennet, Chaulay, Dupuis et Vaillant, à côté de Chachoin, avaient été les secrétaires commis du Comité de rapports de l'Assemblée nationale constituante, mais aussi les secrétaires commis du Comité des pétitions, de la Commission des douze et de la Commission extraordinaire des douze de l'Assemblée législative ${ }^{85}$. Pendant plus de trois ans, la correspondance qui rendait compte des désordres en France et la correspondance qui rendait compte des vœux, des plaintes, des pétitions et des demandes des commettants étaient gérées par les mêmes individus qui poursuivirent leurs travaux, exception faite de Chachoin, jusqu'à la fin de la Convention nationale. Alors que se succédèrent la chute du roi, celle des girondins, celle des hébertistes, celle des dantonistes, celle des robespierristes et celle des hommes de prairial, cinq individus assuraient les mêmes tâches au sein du Comité des pétitions et de correspondance depuis 1789. Mais le Comité des pétitions et de correspondance n'avait pas seulement hérité les secrétaires commis du Comité des rapports, du Comité des pétitions, de la Commission des douze et de la Commission extraordinaire des douze -toujours les mêmes individus-, il avait aussi recruté quelques secrétaires commis qui s'occupaient depuis 1789 de gérer toute la correspondance qui parvenait au Corps législatif, et qui venaient du Bureau des renvois et des correspondances : Atrux, Henry, Leharivel, Aubusson et Renvoizé86. À la différence des secrétaires commis du Comité des rapports, ceux-ci ne travaillèrent d'ailleurs pas toujours sous les ordres du Comité des pétitions et de correspondance. Quand la Commission des dépêches fut créée en avril 1793, elle amena avec elle Aubusson et Thibault, deux secrétaires commis qui étaient chargés à cette époque-là de gérer la correspondance parvenant à l'Assemblée aux ordres du Comité des pétitions et de correspondance ${ }^{87}$. Quand la commission de dépêches fut réunie par décret du 7 fructidor an II au Comité des pétitions et de correspondance, ce dernier récupéra Thibault ainsi que les secrétaires commis qui travaillaient alors dans le cadre de la Commission des dépêches 88. Le Comité des pétitions et de correspondance compta, en conséquence, sur un personnel de base, spécialisé et organisé, capable de mener les tâches exigées au comité et d'encadrer le personnel récemment incorporé ; un personnel qui ne cessa d'augmenter entre 1793 et 1795. Au 4 mai 1793, le comité comptait vingt-deux secrétaires commis et, le 9 prairial an III, il en comptait soixante-trois. En deux ans, son personnel de base avait triplés9. 


\section{Spécialisation. Le secrétaire principal du Comité de salut public}

Ce n'était pas une tâche mineure de décider jour après jour ce qu'on devait lire devant des centaines de députés, un public installé dans les tribunes et une poignée de journalistes prenant note de ce qu'on disait, pas davantage que décider de la correspondance qui devait être adressée à chacun des comités et de déterminer les problèmes qui devaient être résolus par chacun d'entre eux. Ouvrir et lire chacune des lettres qui arrivaient au Corps législatif et décider de son destin, fut probablement une des tâches importantes à accomplir sous une assemblée parlementaire. Adresser un objet à un comité et non pas à un autre pouvait avoir comme conséquence que l'objet fût présenté sous un point de vue particulier. L'importance de la gestion de la correspondance était moins liée à l'efficacité de l'envoi qu'au choix du comité destinataire, ce choix déterminant en grande partie le destin de la lettre. Les décisions cruciales n'étaient pas prises par les membres du Comité des pétitions et de correspondance tous les mercredis et tous les samedis, mais par les membres du comité et adoptées chaque jour avec son personnel de base. Ensemble, ils décidaient de ce qu'on devait lire et de ce qu'on ne devait pas lire à l'Assemblée. Les lettres qu'ils déposaient à la présidence pour être lues par les secrétaires avaient probablement été triées avec soin, elles avaient un contenu politique concret et elles pouvaient provoquer des applaudissements parmi les présents, mais aussi des murmures, voire l'indignation. Cette tâche était si importante que, peu de jours après la mise en place du Comité de salut public, le Comité des pétitions et de correspondance en perdit la gestion au profit de la Commission des dépêches. En fait, depuis 1789 cette tâche avait été assurée, dans un premier temps, par une seule et même personne : le citoyen Aubusson. Depuis le début du processus révolutionnaire, en effet, Aubusson avait la responsabilité d'ouvrir le premier la correspondance qui arrivait et de décider de son sort ${ }^{90}$. En fait, Aubusson était si important qu'au moment où la Commission des dépêches fut mise sur pied il en fit partie, devenant plus tard le chef des secrétaires commis de cette commission. Le 28 juin 1793 la Commission des dépêches décida qu'il aurait la responsabilité de veiller sur la correspondance déposée à la commission et sur le travail de ses confrères :

« Nous députés à la Convention Nationale membres de la Commission des dépêches, ayant reconnu qu'il est indispensable pour le bien et la célérité du service, de confier à l'un des secrétaires commis attachés à notre bureau, connu par son patriotisme, son intelligence et son exactitude (sous sa responsabilité personnelle) le soin et la garde de toutes les pièces et la surveillance du travail de ses confrères, avons nommé pour occuper la place sus dite, en qualité de chef, le citoyen Aubusson, employé dans les bureaux des Assemblées Constituante et Législative, et de la Convention Nationale depuis le premier juillet mil sept-cents quatre vingt neuf, et qui nous a paru réunir les qualités requises pour bien remplir les fonctions importantes que nous jugions nécessaires de lui déléguer. Fait et arrêté à Paris le vingt huit juin mil sept cent quatre vingt treize l'an 2 de la République une et indivisible. Suivent les signatures de Levasseur, Rudel, Christiani, Périès et Guyardin. " ${ }^{91}$

La Commission des dépêches ne fut pas la seule à remarquer le savoir-faire et le talent d'Aubusson dans les tâches qu'il avait assurées depuis le $1^{\text {er }}$ juillet 1789. Le Comité de salut public l'appréciait aussi, étant donné qu'au cours du mois de septembre 1793 il le 
désigna comme secrétaire principal. Et cela parce que Robespierre cherchait un secrétaire de grand mérite :

«Il faut un secrétaire général d'un grand mérite, un Bureau de secrétaires particuliers intelligents et patriotes, des agents également prêts pour transmettre les arrêtés du Comité à ceux qui doivent les exécuter; il faut que dans les vingtquatre heures, ceux qui sont chargés de l'exécution en rendent compte au Comité ; il faut déterminer à qui seront confiés les ordres d'arrêtés, et par qui ils seront transmis; il faut des courriers très sûrs attachés au Comité ; il faut que chaque membre du Comité ait une tâche particulière et qu'il soit entouré de secrétaires et d'agents dignes de sa confiance; il faut que chaque membre ait un emplacement séparé où il puisse travailler et toutes les commodités physiques nécessaires pour agir $[\ldots] »^{92}$

Pour obtenir "une volonté une", et pour que le processus révolutionnaire pût s'accomplir selon une façon déterminée, il fallait aussi des individus intelligents et patriotes travaillant dans les bureaux, capables de gérer tous les réseaux de communications. Aubusson était l'homme que le Comité de salut public cherchait pour la fonction de secrétaire principal, afin de bien organiser les flux d'informations qui circulaient dans ce comité avant de prendre les décisions de défense générale extérieure et intérieure. Il était l'homme qui avait travaillé à l'Assemblée nationale constituante et à l'Assemblée législative sous la direction du président de l'Assemblée, et qui avait travaillé depuis octobre 1792 selon les directives du Comité des pétitions et de correspondance.

\section{Loi. Bulletin national / Bulletin de correspondance}

Henri Grégoire l'avait déjà dit en février 1790 : les désordres avaient lieu parce que les décrets de l'Assemblée n'étaient pas connus ${ }^{93}$. À cette époque-là, il était président du Comité des rapports et à ce titre au courant de tous les désordres en France. Quelques années passèrent, et le 10 août 1792 on remarqua à nouveau combien il était important de faire connaître les décrets de l'Assemblée et «la vérité »/ "les vérités » comme certains disaient. Le Comité des pétitions et de correspondance perdit pendant le mois d'avril des compétences essentielles mais il préserva encore une attribution remarquable, celle d'écrire le Bulletin national. Cet écrit devait unir le peuple à ses représentants en publiant les travaux de la Convention nationale, certains de ses décrets, les lettres des représentants en mission et des généraux, des récits d'actions héroïques et civiques, et les pétitions ainsi que les courriers les plus remarquables des citoyens. Étant donné que la Convention nationale décidait souvent des lettres et des décrets et discours qui devaient être insérés dans le Bulletin national, l'importance du Comité des pétitions et de correspondance dans sa rédaction était due au fait qu'il écrivait les extraits de ces textes et qu'il prenait personnellement note du débat parlementaire. Les rédacteurs du Bulletin national qui faisaient partie du Comité des pétitions et de correspondance occupaient la loge située à la droite du président pour prendre leurs notes ${ }^{94}$, loge qu'ils partageaient avec les rédacteurs du Journal des Débats ${ }^{95}$. Aux membres du Comité des pétitions et de correspondance incombait la tâche de réviser le bulletin, de confirmer ses informations et de les retoucher si nécessaire ${ }^{96}$, tâche qui ne fut pas accordée, apparemment, à Camille Desmoulins quand il fut nommé inspecteur de la section de correspondance ${ }^{97}$.

Le Comité des pétitions et de correspondance fut chargé, entre autres choses, d'écrire un bulletin qui ne pouvait être ni falsifiép8 ni non plus arraché des endroits où il avait été mis à disposition de tous. Ceux qui l'arracheraient seraient punis, la première fois d'une 
amende, la deuxième de deux mois de prison. Ceux qui en empêcheraient la publication seraient punis comme ennemis du peuple et coupables d'offense à la loi ${ }^{99}$. Ce comité non seulement ne perdit pas son contrôle sur le Bulletin national mais, au contraire, il le renforça. Le 3 mai 1793, la Convention nationale accepta sa proposition de faire lui-même la distribution et, à partir de ce moment-là, les ministres de l'Intérieur, de la Guerre et de la Marine cessèrent de le faire. Le comité qui avait vu certaines de ses compétences réduites devenait le responsable de sa distribution et ses secrétaires commis devaient plier les exemplaires sortis de l'imprimerie de Baudouin et préparer les paquets qui devaient être portés à la poste ${ }^{100}$. À partir du mois de mai 1793 , le comité assuma la responsabilité d'adresser le Bulletin national aux départements et aux districts ${ }^{101}$, aux armées de terre et de mer ${ }^{102}$, aux écoles ${ }^{103}$, aux sociétés patriotiques ${ }^{104}$, aux hôpitaux militaires et aux ambulances des armées ${ }^{105}$. À la date du 18 mars 1793 on envoyait 20 exemplaires du Bulletin national à chacun des districts de la République: 15 pour être distribués par le district, 3 pour les écoles et 2 pour les sociétés patriotiques. Quand la distribution du Bulletin national incomba au Comité des pétitions et de correspondance, il lui revint aussi la responsabilité de faire arriver le bulletin ponctuellement à destination. Tous les retards devaient être communiqués au comité par les administrateurs et les généraux. Dans une période où le Bulletin national était la seule manière « officielle » de faire connaître les informations concernant les armées et les travaux de l'Assemblée, la Convention nationale n'acceptait pas de retard dans sa distribution. En juillet 1793, en apprenant que le bulletin n'arrivait pas dans tous les endroits, le Comité des pétitions et de correspondance fut réuni au Comité de sûreté générale pour localiser les causes de ces retards ${ }^{106}$. La mise sur pied du Bulletin des lois le 14 frimaire an II ne déclencha pas la disparition du Bulletin national ${ }^{107}$. D'abord, on établit qu'il s'appellerait Bulletin de correspondance pour le différencier du Bulletin des lois. Ensuite, on décida qu'il ne contiendrait ni les décrets de la Convention ni les récits d'actions héroïques et civiques. Les premiers devaient être publiés dans le Bulletin des lois, les deuxièmes dans le Recueil des belles actions ${ }^{108}$. Néanmoins, le Bulletin de correspondance continua à publier certains décrets quand la Convention le décidait, mais aussi certains rapports des comités et décisions du Comité de salut public et du Comité de sûreté générale, ainsi que des lettres de généraux et de représentants en mission. En définitive, le Bulletin de correspondance ne perdit jamais son importance et avant même que le Bulletin des lois ne fût mis sur pied, le ministre de l'Intérieur avait la responsabilité de le traduire en italien et en allemand ${ }^{109}$. Le 27 septembre 1793, le Comité de salut public décida que le couple Lenscheuring s'occuperait de sa traduction en allemand ${ }^{110}$ et, le 2 octobre 1793, la Société républicaine de Wissembourg demanda sa publication en allemand ainsi qu'en d'autres langues étrangères ${ }^{111}$. À partir du 10 messidor an II (28 juillet 1794), la traduction du Bulletin de correspondance fut gérée par l'Agence de l'envoi des lois. La traduction en allemand fut continuée au-delà de l'activité de la Convention nationale, mais la traduction en italien fut suspendue le 27 germinal an III (16 avril 1795) ${ }^{112}$. Malgré son importance, le Comité des pétitions et de correspondance fut contraint de réduire le nombre d'exemplaires publiés quotidiennement. Autour du mois de nivôse an II (décembre 1794), la Convention nationale estima que les impressions étaient trop coûteuses pour la République et que la distribution qui en était faite était trop considérable. Le 4 nivôse an III, le Comité des pétitions et de correspondance décréta des mesures pour diminuer sa distribution ${ }^{113}$ et demanda à Baudouin, trois jours plus tard, de n'imprimer pour le compte de la République que 22200 exemplaires. 


\section{Recomposition. Le Comité des pétitions, correspondance et dépêches}

Quand Bertrand Barère de Vieuzac prit la parole le 14 thermidor an II pour inviter la Convention nationale à réorganiser les comités et à redéfinir les compétences du Comité de salut public, la troisième métamorphose du Comité des pétitions et de correspondance allait bientôt arriver ${ }^{114}$. La création du Comité de salut public en avril 1793 avait signifié pour le Comité des pétitions et de correspondance la perte de deux compétences importantes ; sa réorganisation signifia, bien au contraire, la restitution d'une de ces deux compétences perdues, le retour du Bureau des renvois et des correspondances. La restitution de ce bureau ne fut pas immédiate, étant donné que le Comité des pétitions et de correspondances n'était pas considéré comme comité à conserver dans le premier rapport que Barère présenta à l'Assemblée. Barère considérait en effet que la Convention nationale devait établir un Comité de salut public, un Comité de sûreté générale et douze comités en relation avec les douze commissions exécutives mises sur pied le 12 germinal an II. En prenant en compte le fait que, selon le rapport de Barère, chaque Comité devait surveiller une commission, il n'y avait pas lieu d'avoir un Comité des pétitions et de correspondance puisqu'il n'y avait aucune Commission exécutive opérant sous ce nom ou ayant les mêmes compétences qui aurait dû être soumise à la surveillance du Comité des pétitions et de correspondance. Néanmoins, dix jours après ce rapport, François-Martin Poultier d'Elmotte signala à la Convention nationale qu'il ne comprenait pas pourquoi le Comité de division et le Comité des dépêches avaient été supprimés.

«Je ne sais par quel motif l'on a supprimé les comités de division et des dépêches, l'un et l'autre me paraissent nécessaires. (...). Le Comité des dépêches est destiné à examiner les pétitions, à les classer, à les analyser, à les renvoyer aux différents comités compétents, à vous faire la lecture des adresses, à vous donner le thermomètre de l'esprit public. Je ne crois pas que l'on puisse supprimer ces comités, puisqu'ils sont nécessaires et qu'ils ne correspondent à aucune des commissions exécutives. $\rrbracket^{115}$

Poultier se référait évidemment à la Commission des dépêches, et non au Comité des pétitions et de correspondance. Néanmoins, trois jours plus tard, le 24 thermidor an II, Théophile Berlier, au nom de la commission établie pour l'organisation des comités, proposa l'établissement d'un Comité des pétitions, correspondance et dépêches, composé de douze membres. L'article dix-neuf de ce projet de décret disait :

«Art. XIX. Le Comité de pétitions, correspondance et dépêches est divisé en 2 sections. La $1^{\text {ère }}$ recueille les pétitions et surveille la composition, l'impression et l'envoi du Bulletin de correspondance. La seconde est chargée de l'ouverture des dépêches, de leur analyse et de la lecture à la tribune. $»^{116}$

Par cet article, la commission établie pour l'organisation des comités demanda à la Convention nationale la mise sur pied du premier Comité des pétitions et de correspondance. Évidemment, sans lui accorder à nouveau la compétence de correspondre avec les représentants en mission, qui restait définitivement attachée au Comité de salut public. La Convention nationale tint compte de cette proposition et établit le 7 fructidor an II un Comité des pétitions, de correspondance et des dépêches ${ }^{117}$. Peut-être, à un moment où les députés cherchaient à voir réduite l'influence du Comité de salut public sur les autres comités, et annulée la soumission des autres comités et commissions à ce comité118, n'y avait-il pas lieu d'avoir une commission de dépêches pour 
adresser au Comité de salut public les informations qui ne pouvaient pas être lues sans inconvénients à l'Assemblée. Le texte suivant fut écrit le 29 avril 1793 :

«Un membre de la Commission des dépêches observe à la Convention Nationale $1^{\circ}$ qu'elle a décrété le 8 de ce mois l'établissement d'une commission chargée spécialement de l'examen des lettres des Ministres, des Généraux, de ses Députés dans les départements et des corps administratifs; de donner la lecture, soit en totalité, soit par extrait de ces différentes pièces et de renvoyer au Comité de salut public les pièces dont la lecture ne pourrait être donnée sans inconvénient ; $2^{\circ}$ que la Convention a décrété le 10 que la Commission des dépêches est autorisée à renvoyer aux divers comités les lettres et pièces énoncées qui n'ont qu'un objet particulier et sur lesquelles elle ne peut statuer sans un rapport. $3^{\circ}$ que les Décrets rendus les 8 et 10 pour l'établissement de cette commission ne se trouvant point insérés dans les procès-verbaux des dits jours, il demande qu'ils y soient rétablis. » 119 comités afin de redéfinir leurs champs d'action, cela n'avait-il guère trop de sens de maintenir la commission de dépêches détachée du Comité des pétitions et de correspondance. En tout cas, pour l'un ou l'autre motif, le Comité des pétitions et de correspondance fut établi définitivement comme un comité attaché surtout à la gestion de la correspondance et à la rédaction du Bulletin national, bien à l'écart des compétences qui avaient permis à ses prédécesseurs de présenter à l'Assemblée les mesures à prendre pour le maintien de l'ordre et de la tranquillité publique. Au mois de fructidor an II, le Comité des pétitions et de correspondance était bien éloigné par ses compétences du Comité de salut public avec qui il partageait une origine commune.

\section{Influence. La voix des commettants, la voix de l'Assemblée}

41 Le pouvoir le plus important du Comité des pétitions et de correspondance fut celui d'influencer à la fois le débat parlementaire et l'opinion publique; d'une part le débat parlementaire par le contrôle qu'il avait de la correspondance parvenant à l'Assemblée il était la voix des commettants-; d'autre part l'opinion publique par le contrôle qu'il avait sur le Bulletin national -il était la voix de l'Assemblée. Le Comité des pétitions et de correspondance ne reçut pas de nombreuses critiques par rapport à ses travaux mais celles qu'il dut affronter concernaient toujours l'influence qu'il pouvait exercer, du fait qu'il pouvait faire connaître un discours au détriment d'un autre. Pour citer un exemple, Jean Féraud, quelques années avant d'être décapité dans les journées de prairial, fut soupçonné de partialité à cause d'un de ses rapports à l'Assemblée ${ }^{120}$. Il fut en effet accusé de n'avoir pas mentionné des pétitions importantes et d'avoir présenté celles qui n'avaient aucune sorte d'intérêt. D'autre part, la rédaction du Bulletin national en date du 30 frimaire an II, provoqua des plaintes à la Convention nationale. François-Louis Bourdon de l'Oise prit la parole pour souligner que le bulletin ne contenait pas la réponse du président de l'Assemblée à une pétition des députés du Club des Cordeliers. De l'avis de ce député, cet oubli ou cette négligence - de la part du Comité des pétitions et de correspondance- risquait de provoquer une interprétation biaisée sur le positionnement de l'Assemblée. Une fois l'intervention de Bourdon finie, quelques députés demandèrent le renouvellement des membres du Comité des pétitions et de correspondance, ainsi que du personnel de base du comitén ${ }^{121}$. Ce renouvellement eut lieu quelques jours plus tard

La Révolution française, 3 | 2012 
mais les membres qui entrèrent ne furent pas le résultat d'une élection ouverte dans le cadre de l'Assemblée, comme cela avait été demandé. Ce ne fut pas non plus le résultat d'une décision unilatérale du Comité de salut public, alors qu'il avait la compétence, depuis le 13 septembre 1793, de choisir les membres qui devaient composer les comités ${ }^{122}$. Les membres qui entrèrent dans le Comité des pétitions et de correspondance à partir du 4 nivôse an II furent choisis par ce même comité, et le Comité de salut public ratifia ce choix ${ }^{123}$. À l'égard de ce renouvellement, on peut considérer que l'intervention du Comité de salut public sur le personnel des autres comités de la Convention nationale ne fut pas aussi interventionniste que Barère le soulignera en thermidor an II ${ }^{124}$.

La pétition pour renouveler le Comité des pétitions et de correspondance, le 30 frimaire an II, ne fut pas isolée. Le 11 vendémiaire an III, un membre fit une nouvelle demande mais la Convention nationale passa à l'ordre du jour ${ }^{125}$, étant donné que son renouvellement devait avoir lieu le 18 vendémiaire an III, conformément au décret du 7 fructidor an II qui le fixait tous les 18 de chaque mois par quart et au moyen d'un scrutin signé. Seuls le Comité de salut public et le Comité de sûreté générale devaient être renouvelés par appel nominal ${ }^{126}$. On peut apprécier l'importance du Comité des pétitions et de correspondance au moment où celui-ci des compétences au profit d'autres comités et quand on demanda son renouvellement, mais aussi quand le Comité de salut public prit la décision de nommer lui-même les députés qui devaient composer la section des pétitions ainsi que ceux pour la section de correspondance ${ }^{127}$. Jusqu'au mois de septembre 1793, c'étaient les membres du comité qui en décidaient eux-mêmes et qui procédaient tous les quinze jours à leur renouvellement. La décision du Comité de salut public d'établir des nominations séparées pour chacune des sections signifiait aussi que les membres chargés de la rédaction du Bulletin national seraient nommés par le Comité de salut public, et non pas par les membres du Comité des pétitions et de correspondance.

Ainsi, occasionnellement centre de toutes les affaires de la Convention, correspondant des commissaires envoyés en mission, organe des corps administratifs et des citoyens de l'Empire, diffuseur de « la vérité » et des décrets de l'Assemblée, porter dans la Convention nationale, et, par ses rapports, thermomètre de l'opinion publique ${ }^{128}$, le Comité des pétitions et de correspondance, héritier du Comité des rapports et cousin germain du Comité de salut public, eut par conséquent une importance considérable sous la Convention nationale d'octobre 1792 à octobre 1795.

\section{NOTES}

1. Ce travail a été réalisé dans le cadre d'une bourse postdoctorale (2008 BP 00149 / Modalitat A del programa Beatriu de Pinós) du gouvernement catalan (Agència de Gestió d'Ajuts Universitaris i de Recerca - AGAUR) et du Grup d'Estudi de les Institucions i de la Societat a la Catalunya Moderna (segles XVI-XIX) (2009-SGR-318).

2. Archives parlementaires des Chambres françaises de 1787 à 1860, recueil complet des débats législatifs et politiques des chambres françaises, première série (1787 à 1799), Paris, Paul Dupont éditeur, 1897, t. 52, p. 280. 
3. Ibid., p. 104.

4. Ibid., p. 232.

5. Ibid., p. 263.

6. Ibid., p. 280.

7. Ibid., p. 263.

8. Ibid., p. 278.

9. Archives parlementaires, op. cit., t. 34, p. 225.

10. Voir Archives nationales (AN), série $D$ « Missions des représentants du peuple et comités des assemblées ", sous la série DXL «Comité des pétitions, dépêches et correspondances ", D*XL 1-5 et registres D*XL 63 et 64 .

11. Le Comité des pétitions reçut 1733 lettres (dossiers) entre janvier et septembre 1792, soit $17,62 \%$ de toute la correspondance enregistrée par le Bureau des renvois et des correspondances. Maria Betlem castellà I Pujols, Revolució, Poder i Informació. El control de la informació a les Assemblees parlamentàries durant la Revolució francesa (1789-1795), thèse de doctorat sous la direction de Lluís Roura i Aulinas et Jean-Clément Martin, Universitat Autònoma de Barcelona et Université de Paris 1 - Panthéon Sorbonne, soutenue à Bellaterra le 28 novembre 2008, t. 2,

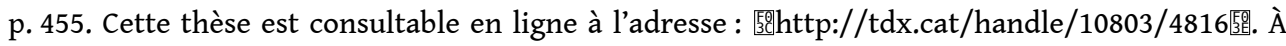
voir également, AN, série $C$ "Enregistrement, procès-verbaux, contrôle, correspondance ", Registre C*II 15 et 16.

12. Archives parlementaires, op. cit., t. 48, p. 304.

13. Ibidem., t. 38, p. 185.

14. Ibid., t. 48, p. 304.

15. Ibid., t. 49 , p. 246.

16. Ibid., t. 49, p. 246.

17. AN, série AF « Archives du pouvoir exécutif (1789-1815) », sous la série AF II « Conseil exécutif provisoire et Convention. Comité de salut public (1792-an IV) », AF II* 18 "Comité des Pétitions et correspondance: procès-verbaux. 1792-an IV ».

18. La section des pétitions du Comité des pétitions et de correspondance était chargée de l'« Enregistrement et [de la] délivrance des numéros aux pétitionnaires qui se présentent au comité pour être admis à la barre. ", AN, AF II* 18 (séance du 16 novembre de l'an I de la République française).

19. Dans le procès-verbal du 16 novembre 1792, le Comité des pétitions et de correspondance annota : "Les citoyens Dechezeau et Feraud ayant fait successivement les rapports dont ils avaient été chargés relativement au Bureau des renvois et de correspondance, le Comité arrête que ce Bureau sera réuni à ceux existant déjà ou qui seront formés pour les diverses parties du travail qui lui est confié pour le décret du onze de ce mois, conformément au plan d'organisation qui sera adopté et lors duquel ces commis qui y sont employés, seront prévenus de la section à laquelle ils seront attachés à moins que les circonstances et le nouvel ordre de travail nécessitent la suppression de quelqu'un d'entre eux. ", AN, AF II* 18 (séance du 16 novembre de l'an I de la République française).

20. Voir les discussions qui eurent lieu à l'Assemblée législative le 21 janvier 1792 et le 4 février de la même année. Archives parlementaires, op. cit., t. 37, p. 577-578 et t. 38, p. 167-171.

21. Ibid., t. 34, p. 510.

22. Maria Betlem CASTELLÀ I PUJOLS, op. cit., p. 440-445.

23. À titre d'exemple, le 14 octobre 1792, Claude Basire prononça le discours suivant : « Le droit de pétition est le droit le plus respectable. Je conjure l'Assemblée de n'y mettre aucune espèce d'entrave, et en conséquence, je demande qu'on ne soumette pas les pétitions à l'arbitraire d'un officier quelconque de l'Assemblée, et que l'on ne décide pas que l'on n'y consacrera qu'une seule séance par semaine. L'objet des pétitions peut quelquefois être très pressant. Ce sont les circonstances qui font naître le besoin des pétitions, et vous ne savez pas comment viendront les 
circonstances. Je demande que les pétitionnaires soient toujours admis à la barre, et que l'Assemblée décrète pour chacun d'eux le moment le plus commode et le plus prompt de les recevoir. ", Archives parlementaires, op. cit., t. 34, p. 217.

24. Ibid., p. 163.

25. Archives Parlementaires, op. cit., t. 34, p. 163.

26. Ibid., p. 159.

27. Cette classification a été établie à partir du travail d'Eduard Resbier sur la communication dans l'entreprise. Eduard RESBIER, «La comunicació a l'empresa», Sociologia de l'empresa, Barcelone, Edicions de l'Universitat de Barcelona, 2003, p. 261-274.

28. Dans le procès-verbal du 24 novembre 1792, le Comité des pétitions et de correspondance annota : «La section de correspondance est chargée particulièrement de la rédaction du bulletin de la Convention nationale et de recueillir les notes et pièces officielles à y insérer, de correspondre avec les commissaires aux armées, de recevoir et expédier les courriers envoyés des départements ou des généraux, de minuter et faire l'envoi des réponses dont le président est chargé ", AN, AF II* 18 (séance du 23 novembre de l'an I de la République française). En ce qui concerne les réponses du président, le Bureau des renvois et des correspondances s'occupait de ce qui suit : "Le même bureau entretient exactement la correspondance de l'Assemblée avec les ministres, les directoires de département et de district, les tribunaux, les municipalités, les gardes nationales, les régiments, généralement avec tous les fonctionnaires publics et même avec les particuliers; soit en composant et expédiant toutes les lettres que l'Assemblée charge son président d'écrire et les réponses que $\mathrm{M}$. le président ordonne de faire aux différentes demandes qui lui sont adressées soit en accusant la réception et en indiquant la destination des pièces qui sont journellement envoyées à l'Assemblée de toutes les parties du royaume. Toutes les lettres décrétées et les réponses importantes sont transcrites sur un registre tenu régulièrement par ordre de dates. Le Bureau de correspondance est encore chargé d'expédier aux départements et aux districts les adresses, discours, rapports et pièces dont l'Assemblée décrète l'impression et l'envoi. » Archives parlementaires, op. cit., t. 30, p. 24.

29. AN, C*II 16.

30. Eduard RESBIER, op. cit., p. 274.

31. Archives parlementaires, op. cit., t. 45, p. 327.

32. Ibidem., t. 48 , p. 348.

33. Henry-Larivière raconta ce qui suit : "Je me suis transporté avec vos commissaires dans les quartiers de Paris où les rassemblements étaient le plus nombreux, et je dois dire, à l'avantage des citoyens de Paris, qu'à peine ils ont reconnu que nous étions leurs représentants, aussitôt la confiance la plus intime, les marques d'amitié la plus tendre, le respect, ont accompagné leurs discours. A l'instant, dis-je, tous se sont tenus découverts et ont entendu la lecture de la loi que nous leur avons faite. Après avoir entendu cette lecture, le peuple s'est livré à une espèce d'enthousiasme, et a dit: "Puisque la loi reprend son empire, puisque enfin le peuple peut être assuré de trouver en elle un appui vraiment fort, nous nous rendons : reportez à l'Assemblée nationale que plutôt que de commettre le crime dont on parle, nous périrons tous !" ", Archives parlementaires, op. cit., t. 47, p. 651. De son côté, Ducos raconta : «Il me paraît que les inquiétudes que les citoyens manifestent viennent de la lenteur avec laquelle on leur a donné connaissance du décret de l'Assemblée nationale. ", Archives parlementaires, op. cit., t. 47, p. 652.

34. Ibidem, t. 47 , p. 645.

35. Ibid., t. 47, p. 642.

36. L'Assemblée décréta : "L'Assemblée nationale, considérant qu'il est nécessaire de faire passer à l'armée, le plus tôt possible, le détail des événements qui ont lieu à Paris, ainsi que la cause de ces événements mêmes, que les circonstances commandent impérieusement d'y apporter la plus grande célérité, décrète qu'il sera nommé à l'instant douze commissaires pris dans son sein pour se rendre sur-le-champ aux diverses armées. ", Archives parlementaires, op. cit., t. 47, p. 659 et 
Michel BIARD, Missionnaires de la République. Les représentants du peuple en mission (1793-1795), Paris, Comité des travaux historiques et scientifiques, 2002, p. 37.

37. Ibid., t. 45, p. 326.

38. Le décret fut le suivant : «L'Assemblée nationale, après avoir décrété l'urgence, décrète qu'il sera mis à la disposition du ministre de l'Intérieur, à la charge d'en rendre compte, la somme de 100000 livres, pour les frais des correspondances qu'il jugera nécessaires, et pour l'impression et distribution, dans les départements et les armées, de tous les écrits propres à éclairer les esprits sur les trames criminelles des ennemis de l'État, et sur les vraies causes des maux qui ont trop longtemps déchiré la patrie. ", Archives parlementaires, op. cit., t. 48, p. 348.

39. Anne KUPIEC, "La Gironde et le Bureau d'esprit public: Livre et Révolution ", Annales historiques de la Révolution française, 1995, n 302, p. 573 et Jacques GUILHAumou, "Décrire la Révolution Française. Les porte-parole et le moment républicain (1790-1793) », Annales: économies, sociétés, civilisations, Paris, 1991, ํㅡ 4, p. 959.

40. Archives parlementaires, op. cit., t. 48, p. 245-246.

41. Anne KUPIEC, art. cit., p. 572.

42. Pierre CARON, La première Terreur (1792). Les missions du Conseil exécutif provisoire et de la Commune de Paris, Paris, Presses universitaires de France, 1950, p. 72.

43. Le discours de Marant qui donna lieu à l'établissement de la Commission extraordinaire des douze le 17 juin 1792 fut le suivant : « Nous ne pouvons plus nous dissimuler les dangers qui nous environnent. Jamais l'État n'eut une marche plus rapide vers sa désorganisation. Jamais la France ne s'est trouvée dans une crise aussi violente et n'a couru autant de dangers. Il est évident que la Constitution n'est pas appliquée, que le pouvoir exécutif se désorganise de lui-même. Il est temps que ce temps d'inquiétude et d'incertitude cesse, il faut que l'Assemblée nationale trouve un moyen de sauver la France ou qu'elle périsse avec elle. Je demande qu'on nomme une nouvelle commission, composée de 12 de ses membres, laquelle sera chargée de lui présenter dans 4 jours, après avoir examiné en général la situation actuelle de l'Empire, le tableau des maux qui désolent la patrie, de leur cause, et des mesures propres à dissiper l'orage qui se forme. ", Archives parlementaires, op. cit., t. 45, p. 326. En outre, le discours de Tardiveau qui donna lieu à l'établissement de la Commission des douze fut ce qui suit : « Depuis plusieurs jours il a été porté au Corps législatif des nouvelles affligeantes. Le peuple, dit-on, égaré par diverses causes s'est porté à des excès, il a servi ainsi, sans le savoir, la cause des ennemis de la patrie. Il convient que l'Assemblée nationale fasse connaître à l'Empire Français que son intention est de maintenir la loi, le respect pour les propriétés, et d'assurer la punition de ceux qui y porteraient atteinte, qu'elle veut que les autorités constituées fassent leur devoir; qu'elle punira celles qui s'en écarteront ; que tous les ministres donneront les ordres nécessaires. ", Archives parlementaires, op. cit., t. 39, p. 426.

44. Ibidem, t. 56, p. 117.

45. Maximilien ROBESPIERRE, CEuvres de Maximilien Robespierre, Paris, Phénix Éditions, 2000, p. 254. Anne KUPIEC, op. cit., p. 584.

46. Archives parlementaires, op. cit., t. 56, p. 112

47. Maria Betlem CASTELLÀ I PUJOLS, op. cit., p. 651-656.

48. AN, AF II*18 (séance du mercredi 30 janvier 1793).

49. Archives parlementaires, op. cit., t. 58, p. 97.

50. Pierre-Charles-Daniel-Gustave Dechézeau, Jean-François Ducos et Pierre Paganel furent choisis pour occuper les trois places réservées au Comité des pétitions et de correspondance dans le cadre du Comité de défense générale, AN, AF II*18 (séance du 31 janvier de l'an II de la République). Voir le tableau no ${ }^{4}$ de l'annexe $n^{\circ} 1$.

51. Françoise BRUNEL, Thermidor. La chute de Robespierre, Bruxelles, Éditions Complexe, 1989, p. 17.

52. Archives parlementaires, op. cit., t. 61, p. 378. 
53. François-Alphonse AULARD, Recueil des actes du Comité de salut public avec la correspondance officielle des représentants en mission et le registre du conseil exécutif provisoire, Paris, Imprimerie nationale, t. III, p. 133.

54. AN, AF II*16 (séance du 29 avril 1793).

55. Dans un document qui a pour titre État des travaux de la Commission des dépêches établi par décret du 8 avril 1793 on peut lire : « Dépositaire des pièces les plus importantes, chargé de recevoir des sommes considérables, que des bons citoyens veulent remettre sur l'autel de la Patrie, le chef du Bureau doit réunir tout ce qui peut fixer la confiance des représentants du peuple, membres de la commission. ", AN, AF II* 16.

56. Dans le document État des travaux de la Commission des dépêches établi par décret du 8 avril 1793 on peut lire : «Les pétitions qui n'offrent aucun objet d'intérêt général sont renvoyées au Comité des Pétitions, après avoir été retenues en note sur un répertoire particulier. » AN, AF II* 16 (sans date). Dans le cadre d'un autre document: "Toutes les lettres, pétitions, mémoires et pièces seront enregistrées et analysées. Ne sont point comprises dans cette disposition, les lettres ou pièces qui n'ayant pour objet que des demandes particulières, seront envoyées au Comité des Pétitions, après cependant avoir été enregistrées par ordre de numéro, sur un registre particulier. ", AN, AF II* 16 (séance du 15 pluviôse an II).

57. Maria Betlem CASTELLÀ I PUJOLS, op. cit., t. 504-508 et 518-519. Voir les tableaux no 1 , no 2 et oํ 3 de l'annexe $\mathrm{n}^{\circ} 1$.

58. Ibidem, p. 516-517. Parmi les 24 premiers députés appelés à composer le Comité de défense général, il y avait 11 députés girondins, 6 députés de la Plaine et 7 députés montagnards. Voir le tableau no 4 de l'annexe $\mathrm{n}^{\circ} 1$.

59. Ibid. p. 525-526. Voir le tableau no 4 de l'annexe $\mathrm{n}^{\circ} 1$.

60. La commission de dépêches fut composée de René Levasseur (Montagne), Marc-FrédéricHenri Christiani (Plaine), Claude-Antoine Rudel (Montagne), Jacques Periès (Gironde) et SimonNicolas Guyardin (Montagne). Maria Betlem CASTELLÀ I PUJOLS, Ibid., p. 526-527.

61. Le Comité de défense générale fut renouvelé le 26 mars 1793, Archives parlementaires, op. cit., t. 60, p. 581. Par le contenu de son procès-verbal, on apprend que le Comité des pétitions et correspondance fut renouvelé entre la fin du mois de mars et le début du mois d'avril, voir AN, AF II*18.

62. En octobre 1792, la liste de députés appelés à composer le Comité des pétitions et de correspondance était composée de 4 députés girondins, 10 députés de la Plaine et 10 députés montagnards. En janvier 1793 la liste était composée de 7 députés girondins, 9 députés de la Plaine et 7 députés montagnards. En avril 1793, la liste était composée de 4 députés girondins, 10 députés de la Plaine et 10 députés montagnards. Néanmoins, indépendamment des listes, le Comité des pétitions et de correspondance était, en raison des absences de ses membres, aux mains des girondins et des députés de la Plaine. D’octobre 1792 à janvier 1793, il était composé de 4 députés girondins, 6 députés de la Plaine et 2 montagnards et, de janvier à mars 1793, il était composé de 4 députés girondins, 4 députés de la Plaine et 4 députés montagnards. Les montagnards n'avaient pas une majorité dans ce comité, néanmoins, ils avaient augmenté leur

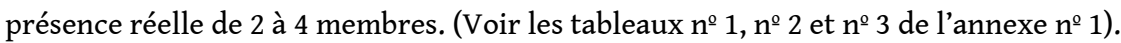

63. Le Comité de défense générale résultant du 26 mars 1793 était composé de 9 députés girondins, 4 députés de la Plaine, 12 députés montagnards. (Voir le tableau ํo 4 de l'annexe ㄲo 1 ). Les références sur les tendances politiques des députés ont été obtenues à partir des travaux suivants : Elie ALLOUCHE, "Comparaison des classifications politiques des conventionnels chez cinq historiens ", Engagements et trajectoires politiques chez les conventionnels. Le problème de la Plaine, mémoire de DEA, sous la direction de Jean-Clément Martin, Université Paris 1 - Panthéon Sorbonne, 2004, Michel BIARD, op. cit., p. 449-592, Jacqueline CHAUMIÉ, «Les girondins », Actes du colloque Girondins et Montagnards, Paris, Société des Études robespierristes, 1980, p. 19-60, Françoise BRUNEL, «Les députés montagnards » et «Liste des 267 députés montagnards en juin 
1793 ", Actes du colloque Girondins et Montagnards, op. cit., p. 343-361 et Albert soBoul, Dictionnaire historique de la Révolution française, Presses universitaires de France, Paris, 1989.

64. Archives parlementaires, op. cit., t. 61, p. 277.

65. Marc bouloiseau, Le Comité de salut public (1793-1795), Paris, Presses universitaires de France, 1968 , p. 18.

66. Archives parlementaires, op. cit., t. 35, p. 584.

67. Archives parlementaires, op. cit., t. 52, p. 280.

68. Ibidem, t. 52, p. 660.

69. Archives parlementaires., Ibidem., t. 53, p. 586.

70. AN, AF II* 18. Voir l'annexe $n^{*} 2$ de cet article. Elle présente tous les députés qui furent élus membres ou suppléants du Comité des pétitions et de correspondance, d'octobre 1792 à octobre 1795 , avec indication de leur assistance aux réunions du comité. Ce tableau a été réalisé à partir des données obtenues dans: $\mathrm{AN}, \mathrm{AF} \mathrm{II}^{*} 18, \mathrm{AD} / \mathrm{XVIIIb}$ : procès-verbaux des assemblées (1787-1875), t. 131-142 et les Archives parlementaires. Nous remercions spécialement Pascal Riviale, conservateur de la série $\mathrm{AD} / \mathrm{XVIIIb}$, de son accord pour la consultation des procès-verbaux imprimés de la Convention nationale entre novembre 1794 et octobre 1795.

71. Archives parlementaires, op. cit., t. 55, p. 354.

72. La Convention nationale adopta le décret suivant: «La Convention nationale décrète que dans trois jours les comités qui, aux termes de son règlement, doivent être renouvelés tous les deux mois, tireront au sort ; que les résultats seront remis aux commissaires de la Salle, qui les feront imprimer sur deux colonnes, celle des membres restants, celle des membres sortis; autorise les comités à mettre sur la liste des sortants ceux qui ne se sont pas rendus avec exactitude à leurs comités : ordonne qu'il sera formé une liste générale de candidats, et qu'il sera ensuite procédé au renouvellement par moitié de chaque comité, suivant l'ordre dans lequel ces comités ont été formés. ", Archives parlementaires, op. cit., t. 55, p. 354.

73. AN, AF II* 18 (séance du samedi 22 décembre an I de la République française). Voir le tableau no 1 de l'annexe no 1 . Dans ce tableau apparaissent indiqués les députés absents du Comité des pétitions et de correspondance à la date du 22 décembre 1793.

74. Archives parlementaires, Ibidem, p. 354.

75. AN, AF II* 18 (séance du samedi 5 janvier 1793). Voir le tableau o 2 de l'annexe oㅡ 1 . Dans ce tableau apparaissent indiqués les députés membres et suppléants du Comité des pétitions et de correspondance en date du 5 janvier 1793, avec indication de ceux qui avaient été membres de ce comité en octobre 1792 et de ceux qui vont rester dans le comité après le renouvellement de celui-ci en avril 1793.

76. Archives Parlementaires, op. cit., t. 78, p. 17-18.

77. Ibid., p. 18.

78. François-Alphonse AULARD, op. cit., t. XVI, p. 311.

79. Voir l'annexe $\mathrm{n}^{\circ} 2$ sur le personnel du Comité des pétitions et de correspondance.

80. AN, AF II*18 (séance du 23 octobre 1792, an I de la République française).

81. Ibid. (séance du 24 vendémiaire an III de la République une et indivisible).

82. Voir le graphique des réunions et l'annexe $n^{\circ} 3$ concernant les réunions (qui furent enregistrées dans les procès-verbaux) du Comité des pétitions et de correspondance. Ce graphique et cette annexe ont été réalisés à partir des procès-verbaux du Comité des pétitions et de correspondance, voir AN, AF II* 18.

83. Rapport du 11 novembre 1792, Archives parlementaires, op. cit., t. 53, p. 355-356 ; rapport du 18 novembre 1792, Ibid., t. 53, p. 463-464 ; rapport du 25 novembre 1792, Ibid., t. 53, p. 577 ; rapport du 2 décembre 1792, Ibid., t. 54, p. 50-52 ; rapport du 9 décembre 1792, Ibid., t. 54, p. 711-713; rapport du 17 décembre 1792, Ibid., t. 55, p. 119-121; rapport du 23 décembre 1792, Ibid., t. 55, p. 365 ; rapport du 31 décembre 1792, Ibid., t. 56, p. 101 ; rapport du 7 janvier 1793, Ibid., t. 56, p. 585-586 ; rapport du 11 janvier 1793, Ibid., t. 56, p. 744-746 ; rapport du 17 février 1793, Ibid., t. 58, 
p. 637-639 ; rapport du 24 février 1793, Ibid., t. 59, p. 131-133 ; rapport du 3 mars 1793, Ibid., t. 59, p. 564-566 ; rapport du 14 mars 1793, Ibid., t. 60, p. 196-198 ; rapport du 22 mars 1793, Ibid., t. 60, p. 445-449; rapport du 24 mars 1793, Ibid., t. 60, p. 516-518 et rapport du 26 mars 1793, Ibid., t. 60, p. 564-565.

84. AN, AF II*18 (séance du 9 prairial an III de l'ère républicaine).

85. Maria Betlem CASTELLÀ I PUJOLS, op. cit., p.486-488 et p. 509-512.

86. Ibid., p. 448-449 et 451 .

87. AN, AF II*16 (séance du 28 juin 1793, an II de la République une et indivisible et séance du 13 septembre 1793, an II de la République une et indivisible).

88. Voir l'annexe $\mathrm{n}^{\mathrm{o}} 2$.

89. Voir l'annexe $\mathrm{n}^{\circ} 4$. Tableau réalisé à propos des "secrétaires commis" du Comité des pétitions et de correspondance. Il a été confectionné à partir des données obtenues dans : AN, AF II*16 et AF II*18, Archives parlementaires et dans un document localisé dans la Collection des documents de Michel Bernstein de l'Université de Senshu à Tokyo. Ce document est intitulé : Liste de MM. les députés composant la Commission extraordinaire des douze et il est daté du 15 août 1792. Le document se trouve dans le tome 58 décrit dans le tome VI du catalogue de la collection : Michel BERNSTEIN, Catalogue de l'histoire de la Révolution française. Comparé avec le catalogue de la Bibliothèque nationale par André Martin et Gérard Walter, Écrits de la période révolutionnaire, supplément aux tomes IV (1) Tomes 1-1766, t. 6. Ikuta, Kawasaki-Shi, Bibliothèque de l'Université de Senshu, 1980.

90. Archives parlementaires, op. cit., t. 30, p. 24.

91. AN, AF II*16 (séance du 28 juin 1793 an II de la République une et indivisible).

92. François FURET et Denis RICHET, La Révolution française [1965], Paris, Fayard, 2002, p. 219.

93. Archives parlementaires, op. cit., t. 11, p. 536.

94. AN, AF II*18 (séance du 3 novembre an I de la République française).

95. Ibid., (séance du 16 novembre an I de la République française).

96. Archives parlementaires, op. cit., t. 78, p. 282.

97. Ibid., p. 282.

98. Dans les Archives parlementaires on peut localiser le texte suivant: «M. Baudouin, qui envoie à l'Assemblée le premier exemplaire du Bulletin national. Il observe que s'il n'était pas signé, des mal intentionnés pourraient le contrefaire pour égarer l'opinion publique; il demande que deux membres de la correspondance signent chaque jour le Bulletin national. », Archives parlementaires, op. cit., t. 49, p. 355.

99. L'article 3 du projet adopté le 15 septembre 1792 était ainsi libellé : « Toute personne qui sera convaincue d'avoir arraché lesdits bulletins, ou empêché leur publication et affiche, sera poursuivie devant les tribunaux, comme ennemie du peuple et coupable d'offense à la loi, à la diligence du procureur de la commune du lieu où seront faites lesdites affiches, et condamnée à 100 livres d'amende pour la première fois, et, en cas de récidive, à deux mois de prison.", Archives parlementaires, op. cit., t. 49, p. 677.

100. Ibidem., t. 64, p. 33-34.

101. La première référence à cette distribution entre les départements et les districts a été localisée dans une lettre de Roland lue le 18 septembre 1792 à l'Assemblée: "L'Assemblée nationale m'a chargé, par le décret qu'elle a rendu, le 15 de ce mois, sur le rapport du Comité de correspondance, d'envoyer à tous les départements et à tous les districts, le bulletin qui s'imprime journellement par les ordres du Corps législatif. Cette mesure est d'une telle importance que je n'ai pas cru devoir attendre l'envoi officiel du décret pour le mettre à exécution. En conséquence, j'ai donné hier par écrit à $M$. Baudouin un état du nombre des bulletins qu'il aura à me remettre tous les matins. ", Archives parlementaires, op. cit., t. 50, p. 107. 102. En janvier 1793, la Convention nationale accepta un projet de décret pour envoyer le Bulletin national plus efficacement aux armées, Archives parlementaires, op. cit., t. 56, p. 724. 
103. Le 23 février 1793, la Convention nationale adopta le décret suivant: «La Convention nationale décrète que son Bulletin sera envoyé à l'avenir aux maisons d'éducation: en conséquence, elle autorise le ministre de l'intérieur à joindre trois exemplaires de ce Bulletin aux quinze qu'il envoie journellement aux districts de la République : elle charge spécialement les directoires de district de la distribution de ces exemplaires aux écoles nationales ", Archives parlementaires, op. cit., t. 59, p. 119.

104. Le 12 mars 1793, la Convention nationale adopta le décret suivant: "La Convention nationale décrète qu'il sera ajouté pour les sociétés patriotiques, deux exemplaires du Bulletin de la Convention, à chaque envoi aux directoires de district, ce qui portera à 20 le nombre de ceux à leur adresser. ", Archives parlementaires, op. cit., t. 60, p. 113.

105. Le 29 mars 1793, la Convention nationale adopta la proposition de Féraud d'envoyer le Bulletin national à tous les hôpitaux militaires ou ambulances des armées. Archives parlementaires, op. cit., t. 60, p. 690 .

106. Archives parlementaires, op. cit., t. 68 , p. 128

107. Ibidem., t. 80, p. 629-630.

108. Ibid., t. 82, p. 398.

109. $\mathrm{AN}, \mathrm{BB}^{29} 278$ : Correspondances de l'agence ou du Bureau de l'envoi des lois et du ministre de la Justice relativement à l'envoi des lois. An II-an IX, p. 166-168.

110. $\mathrm{AN}, \mathrm{BB}^{29} 279$, p. 351-355.

111. Archives parlementaires, op. cit., t. 75, p. 419.

112. AN, $\mathrm{BB}^{29} 278$, p. 296-297.

113. Le 4 nivôse an III, le Comité des pétitions et de correspondance adopta les décisions suivantes: "Primo. Arrête, qu'à compter du 10 du présent mois de nivôse, il n'y aura plus de supplément au Bulletin à moins que des circonstances pressantes ne l'exigent. Secondo. Qu'il ne sera plus inséré au Bulletin aucun rapport des comités sans un décret de la Convention nationale. Tertio. Aucun décret ne sera inséré au Bulletin si la Convention ne l'a pas expressément ordonné. Quarto. Les arrêtés du Comité de salut public et de sûreté générale pour accélérer la publication des mesures et de gouvernement continueront d'y être insérés. Quinto. Aucune adresse, soit des autorités constituées, ou d'individus ne seront insérées au Bulletin sans un décret expresse. Sexto. Aucune des adresses dont la Convention nationale aura décrété l'insertion au bulletin, n'y seront insérées que par extrait, ou par nomenclature, suivant l'importance des objets qu'elles traiteront. Septimo. L'insertion en entier n'aura lieu que lorsqu'un décret l'aura ordonnée. Octavo. Il ne sera plus envoyé qu'un Bulletin aux corps constitués cy après... aux administrateurs du département et de district, aux tribunaux criminels, aux tribunaux de district, aux tribunaux de commerce, aux officiers municipaux de canton, aux juges de paix, aux sociétés populaires, aux comités de surveillance, aux hôpitaux militaires, aux commandants des places, aux administrations d'arrondissement de la Belgique. Nono. Les bulletins continueront à être envoyés aux armées de terre et de mer en nombre cy après aux armées de terre 4560 et aux armées de mer 550. Decimo. La distribution de deux exemplaires à chaque membre de la Convention nationale se fera comme à l'ordinaire. Un décimo. Les bulletins qui seront distribués aux membres de la Convention seront imprimés en octavo. Duodécimo serait pareillement imprimé en octavo ce qui seront adressés aux tribunaux criminels, aux tribunaux de district, aux tribunaux de commerce, aux juges de paix, aux sociétés populaires, aux hôpitaux militaires, aux commandants de places. Tertio decimo. Seront imprimés en placard, ceux qui seront envoyés aux administrations de département, de district, et aux officiers municipaux. Quarto décimo. Il ne sera désormais envoyé de bulletins à aucunes autoritées, soit militaires, soit civiles, autres que celles désignées dans le présent arrêté. », AN, AF II*18 (séance du 4 nivôse an III).

114. Archives parlementaires, op. cit., t. 94, p. 31-34.

115. Ibidem., t. 94, p. 436.

116. Ibid., t. 94, p. 37. 
117. François-Alphonse AULARD, op. cit., t. XVI, p. 311.

118. Le 7 fructidor an II, la Convention nationale décida ce qui suit : «La Convention nationale décrète que les adjonctions des divers comités de la Convention nationale aux Comités de salut public et de sûreté générale, autres que celles déterminées par la loi qui règle les fonctions des Comités, sont supprimées. », François-Alphonse AULARD, Ibidem., t. XVI, p. 310-311.

119. AN, AF II*16 (Extrait du procès-verbal de la Convention nationale du 29 avril 1793, an II de la République française une et indivisible).

120. Ibid., t. 53, p. 464.

121. Ibid., t. 82, p. 69-70.

122. Le 13 septembre 1792, la Convention nationale adopta le décret suivant: "La Convention nationale, sur la proposition d'un membre, décrète que tous les comités, à l'exception de celui de salut public, seront renouvelés; charge son Comité de salut public de lui présenter une liste de candidats pour chacun d'eux. ", Archives parlementaires, op. cit., t. 74, p. 52.

123. Le procès-verbal du Comité des pétitions et de correspondance du 4 pluviôse an II cite ce qui suit: «Le 4 pluviôse l'an 2 de la République française une et indivisible les membres du Comité des pétitions et de correspondance ont arrêté de présenter au Comité de salut public les noms des membres cy-après désignés pour compléter le comité : ces citoyens sont Pellissier, Bassal, Cordier, Bourgain, Lesage-Senault », AN, AF II*18 (séance du 4 pluviôse an II).

124. Le 14 thermidor an II, Barère souligna ce qui suit : «Citoyens, le travail que je suis chargé de vous présenter au nom du Comité de salut public a pour objet une autre délégation de pouvoirs dont la Convention nationale ne doit pas se dessaisir, soit pour l'intérêt de son autorité, soit pour l'intérêt du peuple; cependant, d'après les intentions perfides du dictateur, et par ses manœuvres dans l'Assemblée, il vous avait fait décréter que le Comité de salut public nommerait tous les membres des comités; opération difficile, pénible, odieuse, et tendant à avilir la représentation nationale, et à défavoriser les membres de ce gouvernement aux yeux mêmes de leurs collègues. ", Archives parlementaires, op. cit., t. 94, p. 31. À propos de la compétence que le Comité de salut public avait d'élire les membres des autres comités, Julien Bourdon a écrit ce qui suit : "Même s'il faut remarquer que le Comité de salut public suit souvent les recommandations des anciens membres des Comités, il n'en reste pas moins qu'il est à l'origine de leur composition, la Convention ratifiant sans discussions ses choix. ", Julien BOUdon, Les Jacobins. Une traduction des principes de Jean-Jacques Rousseau, préface de Frédéric Bluche, Paris, L.G.D.J., 2006, p. 504.

125. AN, Ad/XVIIIb/133, p. 230.

126. François-Alphonse AULARD, op. cit., t. XVI, p. 319-320.

127. Archives parlementaires, op. cit., t. 75, p. 180.

128. Cette expression de "Thermomètre de l'opinion publique» fut employée par AnneAlexandre-Marie Thibault le 23 décembre 1792 dans un de ses rapports : « Citoyens, votre Comité des pétitions et correspondance, chargé par un de vos décrets de vous présenter chaque semaine le thermomètre de l'opinion publique, vient vous dire aujourd'hui, par mon organe, que de tous les points de votre Empire les citoyens réunis ou séparés, les assemblées primaires et électorales, les administrations et les tribunaux, les fonctionnaires publics adhèrent avec enthousiasme aux décrets qui abolissent la royauté, et établissent la République une et indivisible. Partout l'amour de la patrie, le feu sacré du civisme le plus pur embrase tous les âmes; on vous jure respect et confiance, et l'on préfère la mort à l'infraction des lois. ", Archives parlementaires, op. cit., t. 55, p. 364. Quelques mois plus tard, le 24 thermidor an II, Poultier parla de « Thermomètre de l'esprit public » pour se référer à la commission de dépêches, Archives parlementaires, op. cit., t. 94, p. 436. 


\section{RÉSUMÉS}

Le Comité des pétitions et de correspondance est un des comités les plus méconnus de l'histoire et de l'historiographie de la Révolution française. Ses antécédents, ses missions et ses compétences n'ont presque jamais attiré l'attention des chercheurs. Trop peu sont ceux qui savent qu'il partage avec le Comité de salut public les mêmes antécédents communs et que la perte de ses compétences en avril 1793 est une conséquence immédiate de l'établissement de ce dernier comité. Parallèlement, on ignore souvent que l'augmentation de ses attributions en novembre 1792 fut faite au détriment de celles du président de l'Assemblée. Peut-être le moment est-il venu de mettre en valeur ce comité et, en même temps, de démontrer qui gérait la correspondance parvenant à l'Assemblée entre 1789 et 1795. Si le contrôle de l'information constitue le quatrième pouvoir, celui-ci ne fut pas absent des assemblées parlementaires et le Comité des pétitions et de correspondance est à cet égard un bon exemple.

The Committee of Petitions and Correspondence is one of the most overlooked committees in the history and historiography of the French Revolution. Its history, missions and competences have almost never come to the attention of researchers. Very few people are aware that it shares a common history with the Committee of Public Safety and that the loss of its competences in April 1793 was an immediate consequence of the creation of the latter. Likewise, it is little known that its competences were increased in November 1792 to the detriment of those of the President of the Assembly. Perhaps the moment has come to highlight this committee and, at the same time, to show the reader who managed the correspondence arriving at the Assembly between 1789 and 1795. If the control of information constitutes the fourth power, then the latter was not absent from parliamentary assemblies, and the Committee of Petitions and Correspondence is, in this respect, a good example.

\section{INDEX}

Mots-clés : Comité des pétitions et de correspondance, président de l'Assemblée, Comité de salut public, information et ordre public

Keywords : Committee of Petitions and Correspondence, President of the Assembly, Committee of Public Safety, Information and Public Order

\section{AUTEUR}

\section{MARIA BETLEM CASTELLÀ I PUJOLS}

Université Paris 1 - Panthéon Sorbonne/

Universitat Pompeu Fabra, Barcelone. 